\title{
Ultra-high pressure direct syntheses of bis(imidazolium-3-yl)alkane dichlorides
}

Article

Accepted Version

Creative Commons: Attribution-Noncommercial-No Derivative Works 4.0

Harwood, L. M., Pitt, P., Scott, J. L. and Sousa, D. (2019) Ultra-high pressure direct syntheses of bis(imidazolium-3yl)alkane dichlorides. Tetrahedron, 75 (44). 130639. ISSN 0040-4020 doi: https://doi.org/10.1016/j.tet.2019.130639 Available at https://centaur.reading.ac.uk/86833/

It is advisable to refer to the publisher's version if you intend to cite from the work. See Guidance on citing.

To link to this article DOI: http://dx.doi.org/10.1016/j.tet.2019.130639

Publisher: Elsevier

All outputs in CentAUR are protected by Intellectual Property Rights law, including copyright law. Copyright and IPR is retained by the creators or other copyright holders. Terms and conditions for use of this material are defined in the End User Agreement.

\section{www.reading.ac.uk/centaur}

\section{CentAUR}

Central Archive at the University of Reading

Reading's research outputs online 


\section{Graphical Abstract}

To create your abstract, type over the instructions in the template box below.

Fonts or abstract dimensions should not be changed or altered.

\section{Ultra-high pressure direct syntheses of}

Leave this area blank for abstract info.

bis(imidazolium-3-yl)alkane dichlorides

L. M. Harwood, ${ }^{\text {a }}$ P. Pitt, ${ }^{\text {a }}$ J. L. Scott, ${ }^{\text {b }}$ and D. Sousa ${ }^{a}$

${ }^{a}$ Department of Chemistry, University of Reading, Whiteknights, Reading, Berkshire, RG6 6AD

${ }^{b}$ Centre for Sustainable Chemical Technologies, 1 South, 1.05, University of Bath, Bath, BA2 7AY

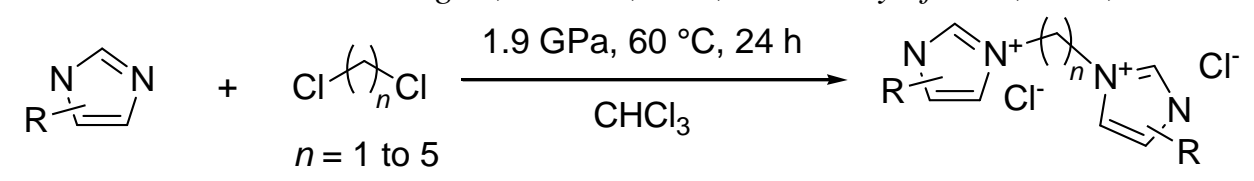

a. 1-methyl-, b. 1-ethyl-, c. 1-butyl-, d. 1,2-dimethyl-,

e. 1,2,4,5-tetramethyl- and f. 1-methyl-2-phenylimidazole

$27-100 \%$ yield, 25 reactions 
Tetrahedron

journal homepage: www.elsevier.com

\title{
Ultra-high pressure direct syntheses of bis(imidazolium-3-yl)alkane dichlorides
}

\author{
Laurence M. Harwood, ${ }^{\mathrm{a} *}$ Phillip Pitt, ${ }^{\mathrm{a}^{*}}$ Janet L. Scott, ${ }^{\mathrm{b}}$ and Dora Sousa ${ }^{\mathrm{a}}$ \\ ${ }^{a}$ Department of Chemistry, University of Reading, Whiteknights, Reading, Berkshire, RG6 6AD \\ ${ }^{b}$ Centre for Sustainable Chemical Technologies, 1 South, 1.05, University of Bath, Bath, BA2 7AY
}

\section{ARTICLE INFO}

\section{Article history:}

Received

Received in revised form

Accepted

Available online

\section{Keywords:}

Ultra-high pressure

Menshutkin reaction

Imidazolium salts

Ionic liquids

Hyperbaric

\section{ABSTRACT}

The ultra-high pressure Menshutkin reaction of $N$-substituted imidazoles and $\alpha, \omega$ dichloroalkanes provides access to a wide variety of bis(imidazoliumyl)alkane dichlorides in an efficient, one-pot process. Although the substitution reactions of dichloromethane at ambient pressure can be exceedingly slow, at $1.9 \mathrm{GPa}$, syntheses are usually quantitative in under $24 \mathrm{~h}$. Reactions involving congested imidazole starting materials, that otherwise terminate after formation of the mono-imidazolium salt, are driven to the bis-imidazolium salt at $1.9 \mathrm{GPa}$. The rates of reaction increase with $\alpha, \omega$-dichloroalkane chain length, decrease with increasing bulk and imidazole $N$-substituent length, and the reactions of the more hindered imidazoles are more sensitive to the pressure-rate enhancement.

2019 Elsevier Ltd. All rights reserved.

\section{Introduction}

As part of an on-going investigation into bis-imidazolium salts atmospherically friendly solvents and particularly ionic liquids, ${ }^{1,2,3,4}$ we herein disclose our findings on the utility of ultra-high pressure (UHP) at $1.9 \mathrm{GPa}$ for the direct synthesis of bis(imidazolium-3-yl)alkane dichlorides via consecutive Menshutkin reactions as illustrated in Scheme 1.

${ }_{\mathrm{Cl}}+\mathrm{r}_{\mathrm{Cl}} \frac{1.9 \mathrm{GPa}, 60{ }^{\circ} \mathrm{C}}{\mathrm{CHCl}_{3}}$

Scheme 1. The one-pot consecutive Menshutkin reaction

UHP conditions enable control over reactions in a manner dependent upon the volume changes associated with different pathways. In accord with Le Chatelier's principle, promoted reactions are those proceeding to species whose formation is accompanied by a decrease in total system volume, be that a transition state for kinetically controlled processes or a product for thermodynamically controlled processes (Fig. 1). ${ }^{5,6}$

The sensitivity of the kinetic response to pressure is quantified by the so-called volume of activation ${ }^{7}\left(\Delta V^{\sharp}\right)$, as defined in (1), where $\Delta V^{*}$ represents the difference in volume between starting materials and the transition state.

$$
\left(\frac{\delta \ln k}{\delta P}\right)_{T}=-\frac{\Delta V^{*}}{R T}
$$

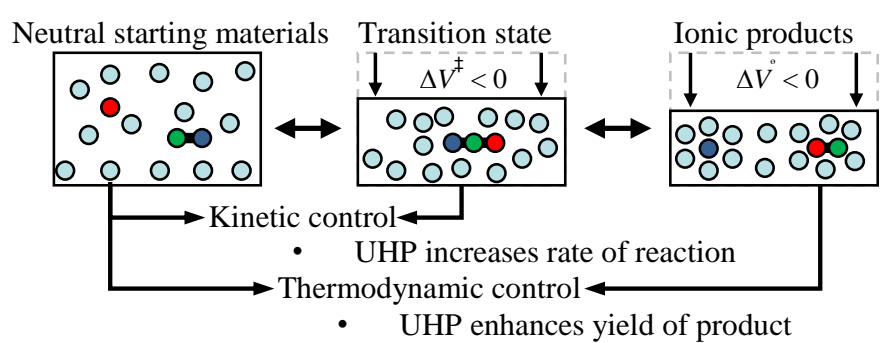

Figure 1. Depiction of volume contractions that lead to the influence of pressure on reactions in liquid media

Ionogenic reactions are particularly strongly promoted by UHP as a result of electostriction ${ }^{8}$ reducing the volume of the solvated species due to the attractive effect that developing charges exert on the solvent. ${ }^{9}$ The Menshutkin synthesis of imidazolium salts from imidazole and dichloroalkanes is intrinsically ionogenic, proceeding through a polar transition state to an ammonium salt product and thus lends itself well to acceleration by the application of UHP..$^{10,11,12}$

Although, the reactivity of dichloromethane towards nucleophilic substitution is generally considered to be low the assumption of complete inactivity, in particular towards $\mathrm{S}_{\mathrm{N}} 2$ reactions, is unwarranted. It has been previously reported that tertiary amines ${ }^{13,14,15}$ and pyridine $^{16}$ react appreciably, albeit slowly, with dichloromethane at ambient temperature and pressure. These reactions have been discovered serendipitously, ${ }^{17}$ for example, in basified HPLC solvents. ${ }^{18}$ 
The preparation of methylene-bridged imidazolium salts from dichloromethane is difficult to achieve with good conversions, although it is sometimes possible to carry out reactions under forcing conditions, such as using a sealed bomb at elevated temperatures. It is more common for dichloromethane to be replaced with the appreciably more reactive dibromomethane or diiodomethane, thus necessitating a subsequent anion metathesis to obtain the chloride salt. Unfortunately, these procedures too can fail for more sterically hindered nucleophiles, such as 1methyl-2-phenylimidazole.

Such compounds have received recent attention for their utility as high-performance anti-wear additives ${ }^{19}$ for use under extreme conditions. For example, in space and aeronautical niches, where elevated temperature and exceedingly low pressures are routine, ionic-liquids as viscous, and practically non-volatile, substances are well-suited ${ }^{20}$ Some of those reported by Yao and co-workers, based on bis(1,2dimethylimidazoliumyl)decane, were found to exhibit excellent tribological characteristics. ${ }^{21}$ These same bis-imidazolium salts belong to the relatively new family of gemini surfactants, ${ }^{22}$ with some examples being at least three-orders of magnitude more effective at reducing surface tension, and two-orders more efficient at forming micelles, than mono-imidazolium salts. ${ }^{23}$ To investigate the properties of these compounds, we pursued a direct and universal synthesis.

\section{Results and Discussion}

A range of symmetrical bis(imidazoliumyl)alkane dichlorides was synthesised from the corresponding imidazoles and $\alpha, \omega$ dichloroalkanes (Scheme 1, Table 1). Whilst at ambient pressure dichloromethane was essentially unreactive towards any of the imidazoles, at $1.95 \mathrm{GPa}$ some reactions were approaching completion within $24 \mathrm{~h}$. Except for the reaction with dichloromethane, 1-methylimidazole reacted extremely rapidly with homologous $\alpha, \omega$-dichloroalkanes at $1.95 \mathrm{GPa}$, with most approaching completion within 3 hours. Reactions of more congested imidazoles were slower, but generally complete within 2-3 days at $1.95 \mathrm{GPa}$.

Increasing the degree of substitution of and hence steric hindrance around the imidazole nucleus resulted in decreased reaction rates with all $\alpha, \omega$-dichloroalkanes, as is to be expected of bimolecular reactions. For example, rates decreased in the order 1-methylimidazole > 1,2-dimethylimidazole > 1,2,4,5tetramethylimidazole > 1-methyl-2-phenyl imidazole, with all dichloroalkanes.

In a consecutive sequence, the first step yields the chloroalkyl mono-imidazolium salt, and the second step yields the bis(imidazolium) product. Reflecting this sequence prior to reaction completion, a time dependent product distribution was obtained for all $\alpha, \omega$-dichloroalkanes other than dichloromethane. An example of a typical reaction progression is illustrated in Figure 2. Relative to the ambient pressure reaction, at $1.95 \mathrm{GPa}$, product distributions were shifted towards the bis-adduct. For reactions of dichloromethane however, the bis(imidazolium-3yl)methane dichloride was the sole product observed.

In general, the maximum percentages of the intermediate mono-imidazolium salt were low and especially so for reactions of the longer dichloroalkanes. The largest percentages were obtained for reactions of 1,2-dichloroethane, and decreased with increasing chain length - the maximum for this transient species decreased and occurred later in the reaction progress as the dichloroalkane chain length was extended (Fig. 3). In contrast, at ambient pressure, the reaction of 1-methyl-2-phenylimidazole with $\alpha, \omega$-dichloroalkanes produced significant quantities of the mono-imidazolium salt and, without application of UHP, the reaction effectively ended after the first substitution step.

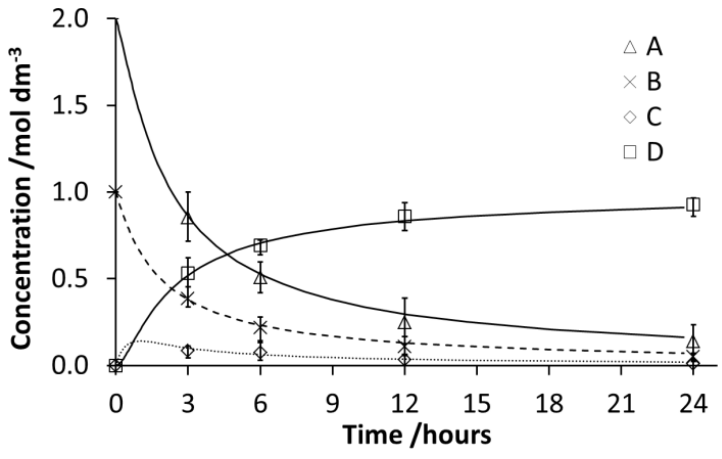

Figure 2. Progression of reaction of 1,2-dimethylimidazole and 1,3-dichloropropane at $1.95 \mathrm{GPa}$ and $45^{\circ} \mathrm{C}$, where A, B, $\mathrm{C}$ and $\mathrm{D}$ are the concentrations of the imidazole, dichloropropane, the mono-imidazolium salt and the bis(imidazolium) salt, respectively.

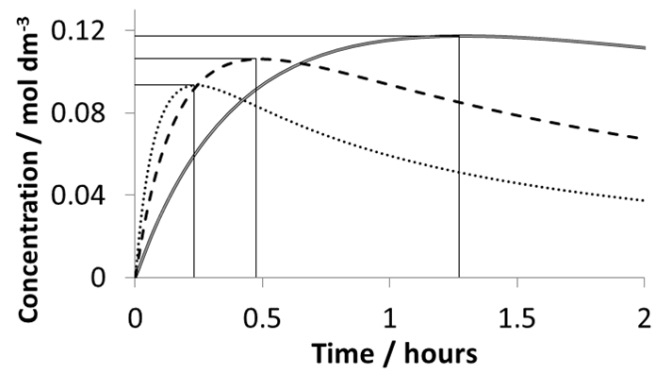

Figure 3. Variation in the early concentrations of monoimidazolium salt for reactions of 1,2-dimethylimidazole with dichlorobutane (dotted line), dichloropropane (dashed line) and dichloroethane (solid line)

The rate constants observed for the reaction of 1,2dimethylimidazole with several dichloroalkanes were estimated using the numerical solution to the differential equations and are listed in Table 2.

Table 2. Rate constants for the reactions of 1,2dimethylimidazole

\begin{tabular}{lll}
\hline 1,2-dimethylimidazole & $k_{1} / \mathrm{mol}^{-1} \mathrm{dm}^{3} \mathrm{~s}^{-1}$ & $k_{2} / k_{1}$ \\
\hline 1,2-dichloroethane & $4.5 \pm 0.6 \times 10^{-5}$ & $5.9 \pm 0.9$ \\
1,3-dichloropropane & $8.8 \pm 1.6 \times 10^{-5}$ & $7.1 \pm 0.4$ \\
1,4-dichlorobutane & $4.2 \pm 0.4 \times 10^{-4}$ & $7.6 \pm 0.7$
\end{tabular}

The enhanced proclivity of the reactions to favour the bis(imidazolium) product at UHP was apparent for every reaction in this study, but was particularly obvious for the reactions of 1methyl-2-phenylimidazole which, as stated above, yielded no bis(imidazolium) salt at ambient pressure. This effect has also been reported for the UHP-promoted consecutive Menshutkin reactions of cyclic amines, including pyrrolidines, piperidines and morpholines with dichloromethane, ${ }^{24}$ and pyridine with $\alpha, \omega$ dichloroalkanes. $^{25}$ For species involved in the Menshutkin reaction, electrostriction increases as the solutes become charged and this will be enhanced upon going from the monoimidazolium adduct to the bis-imidazolium adduct. Indeed, for reactions of tertiary amines with dihaloalkanes, ${ }^{25}$ this has been correlated with the even greater degree of electrostriction 
Table 1. Results for reactions of $N$-substituted imidazoles with various dichloroalkanes in chloroform, using conditions, A: $1.95 \mathrm{GPa}, 60{ }^{\circ} \mathrm{C}$; or B: $1 \mathrm{~atm}$, reflux

\begin{tabular}{|c|c|c|c|c|}
\hline \multirow[b]{2}{*}{ Imidazole } & \multirow[b]{2}{*}{$\alpha, \omega$-dichloroalkane } & \multirow[b]{2}{*}{ Conditions } & \multicolumn{2}{|c|}{ Isolated product yields } \\
\hline & & & of bis-imidazolium salt & of mono-imidazolium salt \\
\hline \multirow[t]{10}{*}{ 1-methylimidazole } & \multirow[t]{2}{*}{ dichloromethane } & $\mathrm{A}$ & $100 \%(24 \mathrm{~h})$ & $0 \%(120 \mathrm{~h})$ \\
\hline & & $\mathrm{B}$ & $0 \%(120 \mathrm{~h})$ & $0 \%(120 \mathrm{~h})$ \\
\hline & \multirow[t]{2}{*}{ 1,2-dichloroethane } & A & $95 \%(24 \mathrm{~h}), 70 \%(3 \mathrm{~h})$ & $2 \%$ \\
\hline & & $\mathrm{B}$ & $77 \%(24 \mathrm{~h})$ & $11 \%(24 \mathrm{~h})$ \\
\hline & \multirow[t]{2}{*}{ 1,3-dichloropropane } & A & $93 \%(3 \mathrm{~h}),>99 \%(24 \mathrm{~h})$ & $1 \%(3 \mathrm{~h}), 0 \%(24 \mathrm{~h})$ \\
\hline & & $\mathrm{B}$ & $83 \%(24 \mathrm{~h})$ & $7 \%$ \\
\hline & \multirow[t]{2}{*}{ 1,4-dichlorobutane } & A & $>99 \%(3 \mathrm{~h})$ & $0 \%(3 \mathrm{~h})$ \\
\hline & & B & $85 \%(24 \mathrm{~h})$ & $2 \%(24 \mathrm{~h})$ \\
\hline & \multirow[t]{2}{*}{ 1,5-dichloropentane } & A & $>99 \%(3 \mathrm{~h})$ & $0 \%(3 \mathrm{~h})$ \\
\hline & & $\mathrm{B}$ & $96 \%(24 \mathrm{~h})$ & $5 \%(24 \mathrm{~h})$ \\
\hline \multirow[t]{8}{*}{ 1-ethylimidazole } & \multirow[t]{2}{*}{ dichloromethane } & A & $76 \%(24 \mathrm{~h})$ & $0 \%(24 \mathrm{~h})$ \\
\hline & & $\mathrm{B}$ & $0 \%(120 \mathrm{~h})$ & $0 \%(120 \mathrm{~h})$ \\
\hline & \multirow[t]{2}{*}{ 1,2-dichloroethane } & A & $87 \%(24 \mathrm{~h})$ & $8 \%(24 \mathrm{~h})$ \\
\hline & & $\mathrm{B}$ & $33 \%(24 \mathrm{~h})$ & $17 \%(24 \mathrm{~h})$ \\
\hline & \multirow[t]{2}{*}{ 1,3-dichloropropane } & A & $95 \%(24 \mathrm{~h})$ & $2 \%(24 \mathrm{~h})$ \\
\hline & & $\mathrm{B}$ & $47 \%(24 \mathrm{~h})$ & $11 \%(24 \mathrm{~h})$ \\
\hline & \multirow[t]{2}{*}{ 1,4-dichlorobutane } & A & $>99 \%(24 \mathrm{~h})$ & $0 \%(24 \mathrm{~h})$ \\
\hline & & $\mathrm{B}$ & $54 \%(24 \mathrm{~h})$ & $7 \%(24 \mathrm{~h})$ \\
\hline \multirow[t]{8}{*}{ 1-butylimidazole } & \multirow[t]{2}{*}{ dichloromethane } & $\mathrm{A}$ & $62 \%(24 \mathrm{~h})$ & $0 \%(24 \mathrm{~h})$ \\
\hline & & $\mathrm{B}$ & $0 \%(120 \mathrm{~h})$ & $0 \%(120 \mathrm{~h})$ \\
\hline & \multirow[t]{2}{*}{ 1,2-dichloroethane } & A & $27 \%(3 \mathrm{~h}), 67 \%(24 \mathrm{~h})$ & $4 \%(24 \mathrm{~h})$ \\
\hline & & $\mathrm{B}$ & $20 \%(24 \mathrm{~h})$ & $7 \%(24 \mathrm{~h})$ \\
\hline & \multirow[t]{2}{*}{ 1,3-dichloropropane } & A & $51 \%(3 \mathrm{~h}), 82 \%(24 \mathrm{~h})$ & $11 \%(3 \mathrm{~h}), 1 \%(24 \mathrm{~h})$ \\
\hline & & B & $31 \%(24 \mathrm{~h})$ & $10 \%(24 \mathrm{~h})$ \\
\hline & \multirow[t]{2}{*}{ 1,4-dichlorobutane } & A & $70 \%(3 \mathrm{~h}), 95 \%(24 \mathrm{~h})$ & $9 \%(3 \mathrm{~h}), 2 \%(24 \mathrm{~h})$ \\
\hline & & $\mathrm{B}$ & $38 \%(24 \mathrm{~h})$ & $8 \%(24 h)$ \\
\hline \multirow[t]{8}{*}{ 1,2-dimethylimidazole } & \multirow[t]{2}{*}{ dichloromethane } & $\mathrm{A}$ & $80 \%(24 \mathrm{~h})$ & $0 \%(24 \mathrm{~h})$ \\
\hline & & $\mathrm{B}$ & $0 \%(120 \mathrm{~h})$ & $0 \%(120 \mathrm{~h})$ \\
\hline & \multirow[t]{2}{*}{ 1,2-dichloroethane } & A & $33 \%(3 \mathrm{~h}), 84 \%(24 \mathrm{~h})$ & $15 \%(3 \mathrm{~h}), 2 \%(24 \mathrm{~h})$ \\
\hline & & B & $20 \%(24 \mathrm{~h})$ & $8 \%(24 h)$ \\
\hline & \multirow[t]{2}{*}{ 1,3-dichloropropane } & A & $53 \%(3 \mathrm{~h}),>99 \%(24 \mathrm{~h})$ & $14 \%(3 \mathrm{~h}), 0 \%(24 \mathrm{~h})$ \\
\hline & & B & $29 \%(24 \mathrm{~h})$ & $11 \%$ \\
\hline & 1,4-dichlorobutane & A & $60 \%(3 \mathrm{~h}),>99 \%(24 \mathrm{~h})$ & $9 \%(3 \mathrm{~h}), 0 \%(24 \mathrm{~h})$ \\
\hline & & $\mathrm{B}$ & $47 \%(24 \mathrm{~h})$ & $13 \%$ \\
\hline $1,2,4,5-$ & dichloromethane & $\mathrm{A}$ & $73 \%(24 \mathrm{~h})$ & $0 \%(24 \mathrm{~h})$ \\
\hline & & B & $0 \%(120 \mathrm{~h})$ & $0 \%(120 \mathrm{~h})$ \\
\hline & 1,2-dichloroethane & A & $60 \%(3 \mathrm{~h}), 80 \%(24 \mathrm{~h})$ & $17 \%(3 \mathrm{~h}), 10 \%(24 \mathrm{~h})$ \\
\hline & & B & $18 \%(24 \mathrm{~h})$ & $17 \%$ \\
\hline & 1,3-dichloropropane & A & $93 \%(24 \mathrm{~h})$ & $0 \%(24 \mathrm{~h})$ \\
\hline & & B & $55 \%(24 \mathrm{~h})$ & $18 \%$ \\
\hline & 1,4-dichlorobutane & A & $>99 \%(3 \mathrm{~h})$ & $0 \%(3 \mathrm{~h})$ \\
\hline & & $\mathrm{B}$ & $66 \%(24 \mathrm{~h})$ & $8 \%(3 \mathrm{~h})$ \\
\hline 1-methyl-2- & dichloromethane & A & $27 \%(24 \mathrm{~h})$ & $0 \%(24 \mathrm{~h})$ \\
\hline & & B & $0 \%(120 \mathrm{~h})$ & $0 \%(120 \mathrm{~h})$ \\
\hline
\end{tabular}




$\begin{array}{ll}\text { 1,2-dichloroethane } & \text { A } \\ \text { 1,3-dichloropropane } & \text { B } \\ & \text { A } \\ \text { 1,4-dichlorobutane } & \text { B } \\ & \text { A } \\ & \text { B }\end{array}$

-associated with the second transition state.

Hence, $\Delta V_{2}^{\neq}$for the second substitution is more negative than $\Delta V_{1}^{\neq}$for the first substitution and the effect of pressure is to increase $k_{2}$ more than $k_{1}$, promoting the second step rate to a larger degree than the first and causing a diminution of the amount of mono-imidazolium intermediate observed.

For reactions of 1,2-dichloroethane, 1,3-propane and 1,4butane this holds true but, by this reasoning, the activation barrier should be greatest for dichloromethane; the results however do not reflect this. The second alkylation step for the reaction of dichloromethane differs to those of the homologous $\alpha, \omega$ dichloroalkanes in that the cationic imidazolium group is geminal to the leaving group. The cationic nature of the 1chloromethylimidazolium intermediate will therefore increase the electrophilic character of the methylene carbon. This effect, in addition to that derived from the pressure-rate enhancement, appears to accelerate the second step to a point where the indeterminate monoalkylation product is not detectable. A similar situation reported for the reaction of pyridine with dichloromethane, wherein no intermediate mono-adduct was detected ${ }^{25}$ was also ascribed to a significantly larger second rate constant.

Whilst at UHP, all reactions proceeded at roughly comparable rates, at ambient pressure reactions of more hindered imidazoles were very slow. Thus, a greater increase in reaction rate on application of UHP is associated with increasingly hindered nucleophiles. This sensitivity to pressure can be rationalised with application of the Hammond postulate. ${ }^{26}$ On affording the transition state, excessive crowding of the reaction centre may cause an increased elongation of the electrophile-nucleofuge bond in the transition state. ${ }^{27}$ This will tend to increase the polarity and charge-separation, to which the activation volume is related. ${ }^{28}$ Subsequently, an increased steric hindrance causes the transition state to occur later along the reaction coordinate, ${ }^{29}$ more closely resembling the cationic product and being in receipt of increased stabilisation due to electrostriction. ${ }^{10,11}$ Conversely, an early transition state is consistent with contemporary analysis of the Menshutkin reaction ${ }^{30}$ providing leeway for the transition state position to be delayed. ${ }^{31}$

The reactions with dichloromethane of five imidazoles were characterised more thoroughly. Over $24 \mathrm{~h}$ reactions were monitored periodically and the concentration versus time profiles fitted using the integrated rate expression are provided in Table 3.

Table 3. Bimolecular rate constants, $k_{1}$, for reactions of $N$ substituted imidazoles with dichloromethane, at $45{ }^{\circ} \mathrm{C}$ and $1.94 \mathrm{GPa}$

\begin{tabular}{ll}
\hline$N$-imidazole & $k_{1} / \mathrm{mol}^{-1} \mathrm{dm}^{3} \mathrm{~s}^{-1}$ \\
\hline 1 -methylimidazole & $2.1 \times 10^{-5} \pm 3.5 \times 10^{-6}$ \\
1 -butylimidazole & $1.1 \times 10^{-5} \pm 2.0 \times 10^{-6}$ \\
1,2 -dimethylimidazole & $1.7 \times 10^{-5} \pm 9.0 \times 10^{-6}$
\end{tabular}

\begin{tabular}{ll}
$33 \%(24 \mathrm{~h})$ & $30 \%(24 \mathrm{~h})$ \\
$0 \%(24 \mathrm{~h})$ & $0 \%(24 \mathrm{~h})$ \\
$60 \%(24 \mathrm{~h})$ & $22 \%(24 \mathrm{~h})$ \\
$0 \%(24 \mathrm{~h})$ & $0 \%(24 \mathrm{~h})$ \\
$71 \%(24 \mathrm{~h})$ & $16 \%(24 \mathrm{~h})$ \\
$0 \%(120 \mathrm{~h})$ & $14 \%(120 \mathrm{~h})$ \\
\multicolumn{1}{c}{$1,2,4,5$-tetramethylimidazole } & $1.4 \times 10^{-5} \pm 1.7 \times 10^{-6}$ \\
1-methyl-2-phenylimidazole & $2.2 \times 10^{-6} \pm 4.0 \times 10^{-7}$
\end{tabular}

\section{Conclusion}

In summary, the ultrahigh pressure Menshutkin reaction provides a convenient means of synthesising bis(imidazolium) dichloride salts, in generally high yield in under $24 \mathrm{~h}$. The method is particularly amenable to support the reactions of hindered imidazoles that do not proceed at acceptable rates at ambient pressure.

\section{Experimental section}

Ultra-high pressure reactions were performed with a Psika Pressure Systems Ltd. (UK) high-pressure hydraulic press using $5 \%$ methanol in castor oil as transmission fluid and 5mL PTFE reaction vessels.

${ }^{1} \mathrm{H}-\mathrm{NMR}$ spectra were recorded on a Bruker AMX 400 NMR (400 MHz) spectrometer and a Bruker Avance DPX 250 (250 $\mathrm{MHz}$ ) spectrometer. Signal positions are recorded as chemical shifts $(\delta)$ in parts per million (ppm) and referenced to the residual solvent peak as an internal standard. ${ }^{13} \mathrm{C}-\mathrm{NMR}$ spectra were recorded on the same spectrometers at $100 \mathrm{MHz}$.

Mass spectra $(\mathrm{m} / \mathrm{z})$ were recorded under conditions of electrospray ionization (ESI) on a Thermo Scientific LTQ Orbitrap mass spectrometer.

Infrared spectra were obtained on a Perkin Elmer Spectrum 100 FT-IR Spectrometer with an attenuated total reflection (ATR) accessory featuring either a diamond or zinc selenide $(\mathrm{ZnSe})$ crystal. The software used was Spectrum Express ${ }^{\circledR}$.

\subsection{General Procedure}

A mixture of the imidazole $(50 \mathrm{mmol})$ and the dichloroalkane ( $25 \mathrm{mmol}$ ) was diluted to $25 \mathrm{~mL}$ with chloroform and degassed by ultra-sonication. The stock solution was stored in the freezer between experimental runs. A $4 \mathrm{~mL}$ aliquot of the stock solution was introduced into a clean, dry, $5 \mathrm{~mL}$ PTFE UHP vessel, sealed with a double O-ring stopper and the head-space gas removed by inserting a syringe needle. The reaction was then pressurised to $1.9 \mathrm{GPa}$ at $60{ }^{\circ} \mathrm{C}$. After the requisite reaction time, the apparatus was depressurised and the vessel removed. The stopper was carefully removed (CAUTION! Potential for pressure build-up) and the contents transferred to a round-bottom flask, the UHP vessel was washed with acetonitrile and the washings combined with the bulk of the reaction mixture. The solvents were removed in-vacuo, and the residue dissolved in $\mathrm{D}_{2} \mathrm{O}$ and analysed immediately by ${ }^{1} \mathrm{H}-\mathrm{NMR}$ spectroscopy. For yields see Table 1 .

\subsection{Bis(1-methylimidazolium-3-yl)methane dichloride}

${ }^{1} \mathrm{H}-\mathrm{NMR}\left(250 \mathrm{MHz}, \mathrm{D}_{2} \mathrm{O}\right): \delta 7.69(2 \mathrm{H}, \mathrm{bs}, \mathrm{C}(4 / 5)-H), 7.51(2 \mathrm{H}$, bs, $\mathrm{C}(4 / 5)-H), 6.22\left(2 \mathrm{H}, \mathrm{s}, \mathrm{CH}_{2}\right)$ and $3.88\left(3 \mathrm{H}, \mathrm{s}, \mathrm{NCH}_{3}\right) ;{ }^{13} \mathrm{C}-$ NMR (62.8 MHz D $\left.\mathrm{D}_{2} \mathrm{O}\right): \delta 138.1(\mathrm{CH}), 124.9(\mathrm{CH}), 121.7(\mathrm{CH})$, $58.7\left(\mathrm{CH}_{2}\right)$ and $\left.36.2\left(\mathrm{CH}_{3}\right) . \mathrm{m} / \mathrm{z}(\mathrm{ESI}): 177.1133\left(\mathrm{M}-\mathrm{HCl}_{2}\right\rceil^{+}\right)$, 
213.0787 (M-Cl1 $\left.{ }^{+}\right), \mathrm{C}_{9} \mathrm{H}_{14} \mathrm{~N}_{4}$ requires 177.1135, found 177.1140. IR (powder) v: 3435, 3385, 3079, 3029, 2091, 1739, 1644, 1642, 1580, 1555, 1474, 1454, 1418, 1330, 1289, 1170, 1079, 1017, $871,772,733,680,620,573 \mathrm{~cm}^{-1}$. Anal. Calcd (\%) for $\mathrm{C}_{9} \mathrm{H}_{14} \mathrm{~N}_{4} \mathrm{Cl}_{2} . \mathrm{H}_{2} \mathrm{O}$ (MW 267.16): C, 40.48; H, 6.04; N, 20.97, Found (\%): C, 39.74; H, 6.07; N, 20.62.

\subsection{1,2-Bis(1-methylimidazolium-3-yl)ethane dichloride}

${ }^{1} \mathrm{H}-\mathrm{NMR}\left(250 \mathrm{MHz}, \mathrm{D}_{2} \mathrm{O}\right): \delta 8.64(2 \mathrm{H}, \mathrm{s}, \mathrm{C}(2)-H), 7.38(2 \mathrm{H}, \mathrm{s}$, $\mathrm{C}(4 / 5)-H), 7.31(2 \mathrm{H}, \mathrm{s}, \mathrm{C}(4 / 5)-H), 4.63\left(4 \mathrm{H}, \mathrm{s}, \mathrm{NCH}_{2}\right)$ and 3.77 $\left(6 \mathrm{H}, \mathrm{s}, \mathrm{NCH}_{3}\right) ;{ }^{13} \mathrm{C}-\mathrm{NMR}\left(62.8 \mathrm{MHz} \mathrm{D}_{2} \mathrm{O}\right): \delta 136.8(\mathrm{CH}), 124.5$ $(\mathrm{CH}), \quad 122.1(\mathrm{CH}), 48.7\left(\mathrm{CH}_{2}\right)$ and $36.0\left(\mathrm{CH}_{3}\right) . \mathrm{m} / \mathrm{z}(\mathrm{ESI})$ : 191.1286 $\left(\mathrm{M}-\mathrm{HCl}_{2} 7^{+}\right), 227.0957\left(\mathrm{M}-\mathrm{Cll}^{+}\right), \mathrm{C}_{10} \mathrm{H}_{15} \mathrm{~N}_{4}{ }^{2+}$ requires 191.1296, found 191.1291. IR (powder) v: 3381, 3059, 2929, 2856, 2100, 1738, 1641, 1573, 1561, 1455, 1379, 1342, 1164, $1114,850,773,715,638,619 \mathrm{~cm}^{-1}$. Anal. Calcd (\%) for $\mathrm{C}_{10} \mathrm{H}_{16} \mathrm{~N}_{4} \mathrm{Cl}_{2} .2 \mathrm{H}_{2} \mathrm{O}$ (MW 299.20): C, 40.14; H, 6.74; N, 18.72, Found (\%): C, 39.84; H, 7.50; N, 18.75.

\subsection{1,3-Bis(1-methylimidazolium-3-yl)propane dichloride}

${ }^{1} \mathrm{H}-\mathrm{NMR}\left(250 \mathrm{MHz}, \mathrm{D}_{2} \mathrm{O}\right): \delta 8.67(2 \mathrm{H}, \mathrm{s}, \mathrm{C}(2)-H), 7.39(2 \mathrm{H}, \mathrm{bs}$, $\mathrm{C}(4 / 5)-H), 7.35(2 \mathrm{H}, \mathrm{bs}, \mathrm{C}(4 / 5)-H), 4.19\left(4 \mathrm{H}, \mathrm{t}, J 7.3 \mathrm{~Hz}, \mathrm{NCH}_{2}\right)$, $3.77\left(6 \mathrm{H}, \mathrm{s}, \mathrm{NCH}_{3}\right)$ and $2.39\left(2 \mathrm{H}\right.$, quin, $\left.J 7.2 \mathrm{~Hz}, \mathrm{CH}_{2}\right) ;{ }^{13} \mathrm{C}-\mathrm{NMR}$ $\left(62.8 \mathrm{MHz}, \mathrm{D}_{2} \mathrm{O}\right): \delta 136.7(\mathrm{CH}), 123.8(\mathrm{CH}), 122.0(\mathrm{CH}), 49.1$ $\left(\mathrm{CH}_{2}\right), 35.7\left(\mathrm{CH}_{3}\right)$ and $29.6\left(\mathrm{CH}_{2}\right) . \mathrm{m} / \mathrm{z}(\mathrm{ESI}): 205.1448(\mathrm{M}-$ $\left.\mathrm{HCl}_{2} 7^{+}\right), 241.1201\left(\mathrm{M}-\mathrm{Cl}^{+}\right), \mathrm{C}_{10} \mathrm{H}_{15} \mathrm{~N}_{4} 205.1453$, found 205.1448 . IR (powder) v: 3369, 3072, 3144, 2124, 1724, 1657, 1562, 1457, 1328, 1279, 1166, 1092, 1065, 1021, 857, 832, 763, 709, 636, $618 \mathrm{~cm}^{-1}$. Anal. Calcd (\%) for $\mathrm{C}_{11} \mathrm{H}_{18} \mathrm{~N}_{4} \mathrm{Cl}_{2} .1 .5 \mathrm{H}_{2} \mathrm{O}$ (MW 304.22): C, 43.43; H, 6.96; N, 18.42 Found (\%): C, 43.98; H, 7.54; N, 18.62 .

\subsection{1,4-Bis(1-methylimidazolium-3-yl)butane dichloride}

${ }^{1} \mathrm{H}-\mathrm{NMR}\left(250 \mathrm{MHz}, \mathrm{D}_{2} \mathrm{O}\right): \delta 8.60(2 \mathrm{H}, \mathrm{s}, \mathrm{C}(2)-H), 7.33(2 \mathrm{H}, \mathrm{s}$, $\mathrm{C}(4 / 5)-H), 7.31(2 \mathrm{H}, \mathrm{s}, \mathrm{C}(4 / 5)-H), 4.11\left(4 \mathrm{H}, \mathrm{t}, J 5.2 \mathrm{~Hz}, \mathrm{NCH}_{2}\right)$, $3.75\left(6 \mathrm{H}, \mathrm{s}, \mathrm{NCH}_{3}\right)$, and $1.76\left(4 \mathrm{H}\right.$, quin, $\left.J 5.2 \mathrm{~Hz}, \mathrm{CH}_{2}\right) ;{ }^{13} \mathrm{C}-$ NMR $\left(62.8 \mathrm{MHz} \mathrm{D}_{2} \mathrm{O}\right): \delta 136.31(\mathrm{CH}), 122.6(\mathrm{CH}), 121.9(\mathrm{CH})$, $48.6\left(\mathrm{CH}_{2}\right), 35.6\left(\mathrm{CH}_{3}\right)$ and $26.1\left(\mathrm{CH}_{2}\right) . \mathrm{m} / \mathrm{z}$ (ESI): $219.1603(\mathrm{M}-$ $\left.\mathrm{HCl}_{2} 7^{+}\right), 255.1358\left(\mathrm{M}-\mathrm{Cl}^{+}\right), \mathrm{C}_{12} \mathrm{H}_{20} \mathrm{~N}_{4}$ requires 219.1609, found 219.1604. IR (powder) v: 3376, 3247, 3145, 3061, 2868, 2958, 2107, 1643, 1577, 1562, 1454, 1429, 1388, 1364, 1334, 1291, $\left.1238,1169,1156,937,859,799,695,651,625,607 \mathrm{~cm}^{-1}\right)$. Anal. Calcd (\%) for $\mathrm{C}_{12} \mathrm{H}_{20} \mathrm{~N}_{4} \mathrm{Cl}_{2} 2 \mathrm{H}_{2} \mathrm{O}$ (MW 327.25): C, 44.04; $\mathrm{H}$, $7.39 ; \mathrm{N}, 17.12$ Found (\%); C, 43.67; H, 7.46; N, 16.73.

\subsection{1,5-Bis(1-methylimidazolium-3-yl)pentane dichloride}

${ }^{1} \mathrm{H}-\mathrm{NMR}\left(250 \mathrm{MHz}, \mathrm{D}_{2} \mathrm{O}\right): \delta 8.58(2 \mathrm{H}, \mathrm{s}, \mathrm{C}(2)-H), 7.33(2 \mathrm{H}, \mathrm{bs}$, $\mathrm{C}(4 / 5)-H), 7.30(2 \mathrm{H}, \mathrm{bs}, \mathrm{C}(4 / 5)-H), 4.05\left(4 \mathrm{H}, \mathrm{t}, J 7.1 \mathrm{~Hz}, \mathrm{NCH}_{2}\right)$, $3.75\left(6 \mathrm{H}, \mathrm{s}, J 2.7 \mathrm{~Hz}, \mathrm{NCH}_{3}\right), 1.78\left(4 \mathrm{H}, \mathrm{tt}, J 8.0 \mathrm{~Hz}, \mathrm{CH}_{2}\right)$, and $1.18\left(2 \mathrm{H}, \mathrm{tt}, J 6.2 \mathrm{~Hz}, \mathrm{CH}_{2}\right) ;{ }^{13} \mathrm{C}-\mathrm{NMR}\left(62.8 \mathrm{D}_{2} \mathrm{O}\right): \delta 135.8(\mathrm{CH})$, $123.5(\mathrm{CH}), 122.1(\mathrm{CH}), 49.7\left(\mathrm{CH}_{2}\right), 35.6\left(\mathrm{CH}_{3}\right), 28.6\left(\mathrm{CH}_{2}\right)$ and $22.7\left(\mathrm{CH}_{2}\right) . \mathrm{m} / \mathrm{z}$ (ESI): $233.1753\left(\mathrm{M}-\mathrm{HCl}_{2} 7^{+}\right), 269.1483\left(\mathrm{M}-\mathrm{Cl} 7^{+}\right)$ $\mathrm{C}_{11} \mathrm{H}_{17} \mathrm{~N}_{4}$ requires 233.1759 , found 233.1753. IR (powder) $v$ : 3365, 3261, 3146, 3083, 2975, 2862, 2171, 1790, 1654, 1576, 1566, 1450, 1425, 1373, 1341, 1272, 1221, 1182, 1082, 984, 898, 841, 793, 745, 651, $623 \mathrm{~cm}^{-1}$. Anal. Calcd (\%) for $\mathrm{C}_{13} \mathrm{H}_{22} \mathrm{~N}_{4} \mathrm{Cl}_{2} .2 \mathrm{H}_{2} \mathrm{O}$ (MW 341.28): C, 45.75; H, 7.68; N, 16.42; Found (\%): C, 45.16; H, 8.17; N, 16.32.

\subsection{Bis(1,2-dimethylimidazolium-3-yl)methane dichloride}

${ }^{1} \mathrm{H}-\mathrm{NMR}\left(250 \mathrm{MHz}, \mathrm{D}_{2} \mathrm{O}\right): \delta 7.48(2 \mathrm{H}, \mathrm{bs}, \mathrm{C}(4 / 5)-H), 7.39(2 \mathrm{H}$, bs, $\mathrm{C}(4 / 5)-H), 6.47\left(2 \mathrm{H}, \mathrm{s}, \mathrm{NCH}_{2}\right), 3.73\left(6 \mathrm{H}, \mathrm{s}, \mathrm{NCH}_{3}\right), 2.66(6 \mathrm{H}$, $\left.\mathrm{s}, \mathrm{CCH}_{3}\right) ;{ }^{13} \mathrm{C}-\mathrm{NMR}\left(62.8 \mathrm{MHz}, \mathrm{D}_{2} \mathrm{O}\right): \delta 146.2(\mathrm{C}), 123.8(\mathrm{CH})$, $120.5(\mathrm{CH}), 57.2\left(\mathrm{CH}_{2}\right), 35.2\left(\mathrm{CH}_{3}\right)$ and $9.4\left(\mathrm{CH}_{3}\right) ; \mathrm{m} / \mathrm{z}(\mathrm{ESI})$ :

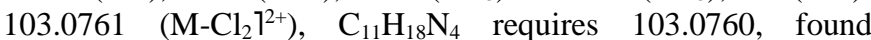

103.0761. IR (powder) v 3390, 3041, 3016, 2960, 1814, 1713, 1592, 1529, 1504, 1443, 1413, 1338, 1299, 1259, 1199, 1139, 1055, 1024, 994, 909, 809, 795, 761, 720, 647, 620, $607 \mathrm{~cm}^{-1}$. Anal. Calcd (\%) for $\mathrm{C}_{11} \mathrm{H}_{18} \mathrm{~N}_{4} \mathrm{Cl}_{2} .0,5 \mathrm{H}_{2} \mathrm{O}$ : (MW 286.20): $\mathrm{C}$, 46.13; H, 6.69; N, 16.50; Found (\%): C, 46.02; H, 6.58; N, 19.50.

\subsection{1,2-Bis(1,2-dimethylimidazolium-3-yl)ethane dichloride}

${ }^{1} \mathrm{H}-\mathrm{NMR}\left(250 \mathrm{MHz}, \mathrm{D}_{2} \mathrm{O}\right): \delta 7.32(2 \mathrm{H}, \mathrm{bs}, \mathrm{C}(4 / 5)-H), 7.16(2 \mathrm{H}$, bs, C(4/5)- $H), 4.55\left(4 \mathrm{H}, \mathrm{s}, \mathrm{NCH}_{2}\right), 3.71\left(6 \mathrm{H}, \mathrm{s}, \mathrm{NCH}_{3}\right), 2.43(6 \mathrm{H}$, $\left.\mathrm{s}, \mathrm{CCH}_{3}\right) .{ }^{13} \mathrm{C}-\mathrm{NMR}\left(62.8 \mathrm{MHz}, \mathrm{D}_{2} \mathrm{O}\right): \delta 145.1(\mathrm{C}), 123.2(\mathrm{CH})$, $120.8(\mathrm{CH}), 46.9\left(\mathrm{CH}_{2}\right), 34.9\left(\mathrm{CH}_{3}\right)$ and $8.6\left(\mathrm{CH}_{3}\right) ; \mathrm{m} / \mathrm{z}(\mathrm{ESI})$ : $110.0840 \quad\left(\mathrm{M}-\mathrm{Cl}_{2} 7^{2+}\right) \quad \mathrm{C}_{12} \mathrm{H}_{20} \mathrm{~N}_{4}$ requires 110.0838 , found 110.0840. IR (powder) v: 3359, 3121, 3044, 2961, 1587, 1536, 1422, 1337, 1282, 1243, 1155, 1125, 1053, 784, 749, 728, 666, $593 \mathrm{~cm}^{-1}$. Anal. Calcd (\%) for $\mathrm{C}_{12} \mathrm{H}_{20} \mathrm{~N}_{4} \mathrm{Cl}_{2} \cdot 3 \mathrm{H}_{2} \mathrm{O}$ : (MW 345.27): C, 41.74; H, 7.58; N, 16.23; Found (\%): C, 41.63; H, 7.02; N, 16.00 .

\subsection{1,3-Bis(1,2-dimethylimidazolium-3-yl)propane dichloride}

${ }^{1} \mathrm{H}-\mathrm{NMR}\left(250 \mathrm{MHz}, \mathrm{D}_{2} \mathrm{O}\right): \delta 7.30(2 \mathrm{H}, \mathrm{bs}, \mathrm{C}(4 / 5)-H), 7.28(2 \mathrm{H}$, bs, C(4/5)-H), $4.15\left(4 \mathrm{H}, \mathrm{t}, J 7.5 \mathrm{~Hz}, \mathrm{NCH}_{2}\right), 3.69\left(6 \mathrm{H}, \mathrm{s}, \mathrm{NCH}_{3}\right)$, $2.51\left(6 \mathrm{H}, \mathrm{s}, \mathrm{CCH}_{3}\right)$ and $2.30\left(2 \mathrm{H}\right.$, quin, $\left.J 7.5 \mathrm{~Hz}, \mathrm{CH}_{2}\right) .{ }^{13} \mathrm{C}-\mathrm{NMR}$ $\left(62.8 \mathrm{MHz}, \mathrm{D}_{2} \mathrm{O}\right): \delta 144.6(\mathrm{C}), 122.6(\mathrm{CH}), 120.4(\mathrm{CH}), 44.8$ $\left(\mathrm{CH}_{2}\right), 34.6\left(\mathrm{CH}_{3}\right), 28.9\left(\mathrm{CH}_{2}\right)$ and $8.8\left(\mathrm{CH}_{3}\right) .{ }^{\mathrm{m}} / \mathrm{z}(\mathrm{ESI}): 117.0918$ $\left(\mathrm{M}-\mathrm{HCl}_{2} 7^{2+}\right), \mathrm{C}_{13} \mathrm{H}_{22} \mathrm{~N}_{4}$ requires 117.0971 , found 117.0918. IR (powder) v: 3349, 3263, 3074, 2970, 2131, 1636, 1587, 1535, 1440, 1377, 1330, 1240, 1191, 1160, 1127, 1048, 879, 808, 766, 752, 727, 670, $658 \mathrm{~cm}^{-1}$. Anal. Calcd (\%) for $\mathrm{C}_{13} \mathrm{H}_{22} \mathrm{~N}_{4} \mathrm{Cl}_{2} \cdot 2 \mathrm{H}_{2} \mathrm{O}$ : (MW 341.28): C, 45.75; H, 7.68; N, 16.42; Found (\%): C, 45.26; $\mathrm{H}, 7.66 ; \mathrm{N}, 16.13$.

\subsection{1,4-Bis(1,2-dimethylimidazolium-3-yl)butane dichloride}

${ }^{1} \mathrm{H}-\mathrm{NMR}\left(250 \mathrm{MHz}, \mathrm{D}_{2} \mathrm{O}\right): \delta 7.23(4 \mathrm{H}, \mathrm{bs}, \mathrm{C}(4 / 5)-H), 4.05(4 \mathrm{H}$, $\left.\mathrm{s}, \mathrm{NCH}_{2}\right), 3.56\left(6 \mathrm{H}, \mathrm{s}, \mathrm{NCH}_{3}\right), 2.47\left(6 \mathrm{H}, \mathrm{s}, \mathrm{CCH}_{3}\right)$ and $1.08(4 \mathrm{H}$, t, $\left.J 7.2 \mathrm{~Hz}, \mathrm{CH}_{2}\right) .{ }^{13} \mathrm{C}-\mathrm{NMR}\left(62.8 \mathrm{MHz}, \mathrm{D}_{2} \mathrm{O}\right): \delta 145.0(\mathrm{C}), 122.3$ $(\mathrm{CH}), 120.5(\mathrm{CH}), 47.4\left(\mathrm{CH}_{2}\right), 34.5\left(\mathrm{CH}_{3}\right), 25.9\left(\mathrm{CH}_{2}\right)$ and 8.7

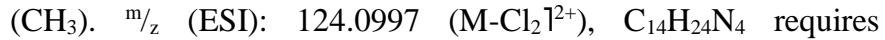
124.0995, found 124.0997. IR (powder) v: 3443, 3381, 3050, 1587, 1536, 1470, 1365, 1341, 1270, 1242, 1152, 1118, 1037, 795, 778, 730, 660, $628 \mathrm{~cm}^{-1}$. Anal. Calcd (\%) for $\mathrm{C}_{14} \mathrm{H}_{24} \mathrm{~N}_{4} \mathrm{Cl}_{2} .1,5 \mathrm{H}_{2} \mathrm{O}$ : (MW 346.30): C, 48.56; H, 7.86; N, 16.18; Found (\%): C, 48.52; H, 8.33; N, 15.65 .

\subsection{1,5-Bis(1,2-dimethylimidazolium-3-yl)pentane dichloride}

${ }^{1} \mathrm{H}-\mathrm{NMR}\left(250 \mathrm{MHz}, \mathrm{D}_{2} \mathrm{O}\right): \delta 7.25(4 \mathrm{H}, \mathrm{bs}, \mathrm{C}(4 / 5)-H), 4.02(4 \mathrm{H}$, t, $\left.J 7.2 \mathrm{~Hz}, \mathrm{NCH}_{2}\right), 3.67\left(6 \mathrm{H}, \mathrm{s}, \mathrm{NCH}_{3}\right), 2.48\left(6 \mathrm{H}, \mathrm{s}, \mathrm{CCH}_{3}\right), 1.74$ (4H, quin, $\left.J 7.6 \mathrm{~Hz}, \mathrm{CH}_{2}\right)$ and $1.27\left(2 \mathrm{H}, \mathrm{m}, \mathrm{CH}_{2}\right) .{ }^{13} \mathrm{C}-\mathrm{NMR}(62.8$ $\left.\mathrm{MHz}, \mathrm{D}_{2} \mathrm{O}\right): \delta 144.2(\mathrm{C}), 122.1(\mathrm{CH}), 120.6(\mathrm{CH}), 47.8\left(\mathrm{CH}_{2}\right)$, $34.6\left(\mathrm{CH}_{3}\right), 28.2\left(\mathrm{CH}_{2}\right), 22.4\left(\mathrm{CH}_{2}\right)$ and $8.8\left(\mathrm{CH}_{3}\right) ; \mathrm{m} / \mathrm{z}(\mathrm{ESI})$ : $131.1073\left(\mathrm{M}-\mathrm{Cl}_{2} 7^{2+}\right), \quad \mathrm{C}_{15} \mathrm{H}_{26} \mathrm{~N}_{4}$ requires 131.1073, found 131.1076. IR (powder) v: 3387, 3036, 2945, 1586 1538, 1514, 1462, 1420, 1391, 1358, 1338, 1274, 1246, 1190, 1122, 1077, 1049, 787, 753, 739, $661 \mathrm{~cm}^{-1}$. Anal. Calcd (\%) for $\mathrm{C}_{15} \mathrm{H}_{26} \mathrm{~N}_{4} \mathrm{Cl}_{2} .4 \mathrm{H}_{2} \mathrm{O}$ : (MW 405.36): C, 44.44; $\mathrm{H}, 8.45 ; \mathrm{N}, 13.82$; Found (\%): C, 43.70; H, 7.12; N, 13.07.

\subsection{Bis(1-ethylimidazolium -3-yl)methane dichloride}

${ }^{1} \mathrm{H}-\mathrm{NMR}\left(400 \mathrm{MHz}, \mathrm{D}_{2} \mathrm{O}\right): \delta 9.16(2 \mathrm{H}, \mathrm{s}, \mathrm{C}(2)-H), 7.66(2 \mathrm{H}, \mathrm{bs}$, $\mathrm{C}(4 / 5)-H), 7.55(2 \mathrm{H}, \mathrm{bs}, \mathrm{C}(4 / 5)-H), 6.59\left(2 \mathrm{H}, \mathrm{s}, \mathrm{NCH}_{2}\right), 4.21$ $\left(4 \mathrm{H}, \mathrm{q}, J 7.2 \mathrm{~Hz}, \mathrm{NCH}_{2} \mathrm{CH}_{3}\right), 1.43\left(6 \mathrm{H}, \mathrm{t}, J 7.2 \mathrm{~Hz}, \mathrm{CCH}_{3}\right) .{ }^{13} \mathrm{C}-$ NMR (62.8 MHz, $\left.\mathrm{D}_{2} \mathrm{O}\right): \delta 135.2(\mathrm{CH}), 123.4(\mathrm{CH}), 122.0(\mathrm{CH})$, $58.8\left(\mathrm{CH}_{2}\right), 45.6\left(\mathrm{CH}_{2}\right)$ and $14.1\left(\mathrm{CH}_{3}\right) ; \mathrm{m} / \mathrm{z}(\mathrm{ESI}): 205.1445(\mathrm{M}-$ $\left.\mathrm{HCl}_{2} 7^{+}\right), 103.0754\left(\mathrm{M}-\mathrm{Cl}_{2} 7^{2+}\right), \mathrm{C}_{11} \mathrm{H}_{17} \mathrm{~N}_{4}$ requires 205.1448 , found 205.1445. IR (powder) v: 3362, 3245, 3040, 2983, 2116, 1642, 1579, 1560, 1544, 1512, 1448, 1396, 1350, 1324, 1228, 1166, 
1109, 1080, 1034, 958, 916, 885, 805, 763, 666, 618, $607 \mathrm{~cm}^{-1}$. Anal. Calcd (\%) for $\mathrm{C}_{11} \mathrm{H}_{18} \mathrm{~N}_{4} \mathrm{Cl}_{2} .1 \mathrm{H}_{2} \mathrm{O}$ : (MW 295.21): C, 44.75; H, 6.82; N, 18.98; Found (\%): C, 43.85; H, 6.76; N, 18.70.

\subsection{1,2-Bis(1-ethylimidazolium-3-yl)ethane dichloride}

${ }^{1} \mathrm{H}-\mathrm{NMR}\left(400 \mathrm{MHz}, \mathrm{D}_{2} \mathrm{O}\right): \delta 8.74(2 \mathrm{H}, \mathrm{s}, \mathrm{C}(2)-H), 7.52(2 \mathrm{H}, \mathrm{bs}$, $\mathrm{C}(4 / 5)-H), 7.36(2 \mathrm{H}, \mathrm{bs}, \mathrm{C}(4 / 5)-H), 4.67\left(4 \mathrm{H}, \mathrm{s}, \mathrm{NCH}_{2}\right), 4.17$ $\left(4 \mathrm{H}, \mathrm{q}, J 7.4 \mathrm{~Hz}, \mathrm{NCH}_{2} \mathrm{CH}_{3}\right), 1.42\left(6 \mathrm{H}, \mathrm{t}, J 7.4 \mathrm{~Hz}, \mathrm{CCH}_{3}\right) .{ }^{13} \mathrm{C}-$ $\operatorname{NMR}\left(62.8 \mathrm{MHz}, \mathrm{D}_{2} \mathrm{O}\right): \delta 135.6(\mathrm{CH}), 123.2(\mathrm{CH}), 122.2(\mathrm{CH})$, $48.8\left(\mathrm{CH}_{2}\right), 45.2\left(\mathrm{CH}_{2}\right)$ and $14.4\left(\mathrm{CH}_{3}\right) ; \mathrm{m} / \mathrm{z}(\mathrm{ESI}): 219.1601(\mathrm{M}-$ $\left.\mathrm{HCl}_{2} 7^{+}\right), 110.0844\left(\mathrm{M}^{-} \mathrm{Cl}_{2} 7^{2+}\right), \mathrm{C}_{12} \mathrm{H}_{19} \mathrm{~N}_{4}$ requires 219.1604, found 219.1601. IR (powder) v: 3378, 3140, 3078, 3031, 2875, 2110, 1639, 1561, 1449, 1409, 1356, 1336, 1302, 1246, 1166, 1122, 962, 864, 799, 785, 652, 638, $603 \mathrm{~cm}^{-1}$. Anal. Calcd (\%) for $\mathrm{C}_{12} \mathrm{H}_{20} \mathrm{~N}_{4} \mathrm{Cl}_{2} .2 \mathrm{H}_{2} \mathrm{O}$ : (MW 327.25): C, 44.04; H, 7.39; N, 17.12; Found (\%): C, 44.48; H, 8.04; N, 17.33.

\subsection{1,3-Bis(1-ethylimidazolium-3-yl)propane dichloride}

${ }^{1} \mathrm{H}-\mathrm{NMR}\left(400 \mathrm{MHz}, \mathrm{D}_{2} \mathrm{O}\right): \delta 8.73(2 \mathrm{H}, \mathrm{s}, \mathrm{C}(2)-H), 7.44(2 \mathrm{H}, \mathrm{bs}$, $\mathrm{C}(4 / 5)-H), 7.41(2 \mathrm{H}$, bs, C(4/5)-H), $4.17(4 \mathrm{H}, \mathrm{t}, J 7.4 \mathrm{~Hz}$, $\left.\mathrm{NCH}_{2} \mathrm{CH}_{3}\right), 4.16\left(4 \mathrm{H}, \mathrm{q}, J 7.4 \mathrm{~Hz}, \mathrm{NCH}_{2}\right), 1.85(2 \mathrm{H}$, quin, $J 4.7$ $\left.\mathrm{Hz}, \mathrm{CH}_{2}\right), 1.44\left(6 \mathrm{H}, \mathrm{t}, J 7.4 \mathrm{~Hz}, \mathrm{CH}_{2} \mathrm{CH}_{3}\right) .{ }^{13} \mathrm{C}-\mathrm{NMR}(62.8 \mathrm{MHz}$, $\left.\mathrm{D}_{2} \mathrm{O}\right): \delta 134.9(\mathrm{CH}), 122.2(\mathrm{CH}), 122.1(\mathrm{CH}), 48.7\left(\mathrm{CH}_{2}\right), 44.9$ $\left(\mathrm{CH}_{2}\right), 26.2\left(\mathrm{CH}_{2}\right), 14.4\left(\mathrm{CH}_{3}\right) ; \mathrm{m} / \mathrm{z}(\mathrm{ESI}): 233.1759\left(\mathrm{M}-\mathrm{HCl}_{2} \mathrm{1}^{+}\right)$,

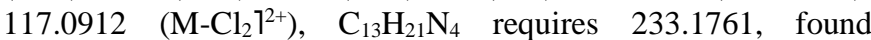
233.1759. IR (powder) v: 3378, 3134, 3048, 2980, 2946, 2866, 2161, 1564, 1460, 1357, 1258, 1156, 1095, 1050, 958, 824, 779, $741,652,618 \mathrm{~cm}^{-1}$. Anal. Calcd $(\%)$ for $\mathrm{C}_{13} \mathrm{H}_{22} \mathrm{~N}_{4} \mathrm{Cl}_{2} \cdot 0.5 \mathrm{H}_{2} \mathrm{O}$ : (MW 323.27): C, 49.69; H, 7.38; N, 17.83; Found (\%): C, 49.32; $\mathrm{H}, 8.93 ; \mathrm{N}, 17.64$

\subsection{1,4-Bis(1-ethylimidazolium-3-yl)butane dichloride}

${ }^{1} \mathrm{H}-\mathrm{NMR}\left(400 \mathrm{MHz}, \mathrm{D}_{2} \mathrm{O}\right): \delta 8.72(2 \mathrm{H}, \mathrm{s}, \mathrm{C}(2)-H), 7.43(2 \mathrm{H}, \mathrm{bs}$, $\mathrm{C}(4 / 5)-H), 7.39(2 \mathrm{H}, \mathrm{bs}, \mathrm{C}(4 / 5)-H), 4.16(4 \mathrm{H}, \mathrm{dd}, J 4.8 \mathrm{~Hz}$, $\left.\mathrm{NCH}_{2} \mathrm{CH}_{3}\right), 4.18\left(4 \mathrm{H}, \mathrm{t}, J 7.6 \mathrm{~Hz}, \mathrm{NCH}_{2} \mathrm{CH}_{2}\right), 1.82(4 \mathrm{H}, \mathrm{q}, J 4.8$ $\left.\mathrm{Hz} \mathrm{CH}_{2} \mathrm{CH}_{2}\right), 1.44\left(6 \mathrm{H}, \mathrm{t}, J 7.2 \mathrm{~Hz}, \mathrm{CH}_{3}\right) .{ }^{13} \mathrm{C}-\mathrm{NMR}(62.8 \mathrm{MHz}$, $\left.\mathrm{D}_{2} \mathrm{O}\right): \delta 135.2(\mathrm{CH}), 122.5(\mathrm{CH}), 122.2(\mathrm{CH}), 46.4\left(\mathrm{CH}_{2}\right), 44.9$ $\left(\mathrm{CH}_{2}\right), 29.6\left(\mathrm{CH}_{2}\right)$ and $14.4\left(\mathrm{CH}_{3}\right) ; \mathrm{m} / \mathrm{z}(\mathrm{ESI}): 247.1915(\mathrm{M}-$

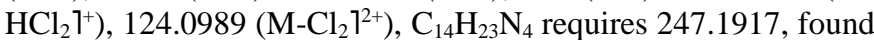
247.1915. IR (powder) v: 3379, 3134, 3075, 2976, 2923, 2828, 1823, 1630, 1556, 1450, 1376, 1304, 1220, 1156, 1055, 1033, 1018, 958, 916, 796, 784, $649 \mathrm{~cm}^{-1}$. Anal. Calcd (\%) for $\mathrm{C}_{14} \mathrm{H}_{24} \mathrm{~N}_{4} \mathrm{Cl}_{2} . \mathrm{H}_{2} \mathrm{O}$ : (MW 337.29): C, 49.85; H, 7.77; N, 16.61; Found (\%): C, 50.42; H, 8.16; N, 16.55 .

\subsection{1,5-Bis(1-ethylimidazolium-3-yl)pentane dichloride}

${ }^{1} \mathrm{H}-\mathrm{NMR}\left(400 \mathrm{MHz}, \mathrm{D}_{2} \mathrm{O}\right): \delta 8.70(2 \mathrm{H}, \mathrm{s}, \mathrm{C}(2)-H), 7.43(2 \mathrm{H}, \mathrm{bs}$, $\mathrm{C}(4 / 5)-H), 7.39(2 \mathrm{H}$, bs, C(4/5)-H), $4.14(4 \mathrm{H}, \mathrm{t}, J 7.2 \mathrm{~Hz}$, $\left.\mathrm{NCH}_{2} \mathrm{CH}_{2}\right), 4.11\left(4 \mathrm{H}, \mathrm{q}, J 7.5 \mathrm{~Hz}, \mathrm{NCH}_{2} \mathrm{CH}_{3}\right), 1.84(4 \mathrm{H}$, quin, $J$ $\left.7.6 \mathrm{~Hz}, \mathrm{CH}_{2}\right), 1.39\left(6 \mathrm{H}, \mathrm{t}, J 7.5 \mathrm{~Hz}, \mathrm{CH}_{2} \mathrm{CH}_{3}\right), 1.22(2 \mathrm{H}$, quin, $J$ $\left.7.5 \mathrm{~Hz}, \mathrm{CH}_{2}\right) .{ }^{13} \mathrm{C}-\mathrm{NMR}\left(62.8 \mathrm{MHz}, \mathrm{D}_{2} \mathrm{O}\right): \delta 134.8(\mathrm{CH}), 122.2$ $(\mathrm{CH}), 122.0(\mathrm{CH}), 49.1\left(\mathrm{CH}_{2}\right), 44.8\left(\mathrm{CH}_{2}\right), 28.7\left(\mathrm{CH}_{2}\right), 22.2$ $\left(\mathrm{CH}_{2}\right), 14.4\left(\mathrm{CH}_{3}\right) ; \mathrm{m} / \mathrm{z}$ (ESI): $261.2071\left(\mathrm{M}-\mathrm{HCl}_{2} 7^{+}\right), 131.1067$ $\left(\mathrm{M}-\mathrm{Cl}_{2} 7^{2+}\right), \mathrm{C}_{15} \mathrm{H}_{25} \mathrm{~N}_{4}$ requires 261.2074, found 261.2071. IR (powder) v: 3442, 3383, 3086, 3045, 2977, 2200, 2122, 1790, $1681,1621,1577,1562,1451,1385,1344,1320,1268,1231$, $1193,1164,1033,876,789,692,650,627,584 \mathrm{~cm}^{-1}$.

\subsection{Bis(1-butylimidazolium-3-yl)methane dichloride}

${ }^{1} \mathrm{H}-\mathrm{NMR}\left(400 \mathrm{MHz}, \mathrm{D}_{2} \mathrm{O}\right): \delta 7.71(2 \mathrm{H}, \mathrm{bs}, \mathrm{C}(4 / 5)-H), 7.59(2 \mathrm{H}$, bs, C(4/5)- $H), 6.63\left(2 \mathrm{H}, \mathrm{s}, \mathrm{NCH}_{2}\right), 4.20(4 \mathrm{H}$, quin, $J 7.6 \mathrm{~Hz}$, $\left.\mathrm{NCH}_{2} \mathrm{CH}_{2}\right), 1.78\left(6 \mathrm{H}\right.$, quin, $\left.J 7.6 \mathrm{~Hz}, \mathrm{CH}_{2} \mathrm{CH}_{2}\right), 1.23(6 \mathrm{H}$, quin, $J$ $\left.7.6 \mathrm{~Hz}, \mathrm{CH}_{2} \mathrm{CH}_{2}\right)$ and $0.82\left(6 \mathrm{H}, \mathrm{t}, J 7.6 \mathrm{~Hz}, \mathrm{CH}_{2} \mathrm{CH}_{3}\right) .{ }^{13} \mathrm{C}-\mathrm{NMR}$ $\left(62.8 \mathrm{MHz}, \mathrm{D}_{2} \mathrm{O}\right): \delta 136.4(\mathrm{CH}), 123.8(\mathrm{CH}), 122.0(\mathrm{CH}), 58.8$ $\left(\mathrm{CH}_{2}\right), 50.1\left(\mathrm{CH}_{2}\right), 31.0\left(\mathrm{CH}_{2}\right), 18.8\left(\mathrm{CH}_{2}\right)$ and $12.9\left(\mathrm{CH}_{3}\right) ; \mathrm{m} / \mathrm{z}$ (ESI): $261.2072\left(\mathrm{M}-\mathrm{HCl}_{2} 7^{+}\right), 131.1068\left(\mathrm{M}^{-\mathrm{Cl}_{2}} 7^{2+}\right), \mathrm{C}_{15} \mathrm{H}_{25} \mathrm{~N}_{4}$ requires 261.2074, found 261.2072. IR (powder) v: 3406, 3051, 2960, 2873, 1794, 1681, 1580, 1560, 1462, 1380, 1320, 1166, $1114,906,802,768,717,665,612 \mathrm{~cm}^{-1}$.

\subsection{1,2-Bis(1-butylimidazolium-3-yl)ethane dichloride}

${ }^{1} \mathrm{H}-\mathrm{NMR}\left(400 \mathrm{MHz}, \mathrm{D}_{2} \mathrm{O}\right): \delta 8.79(2 \mathrm{H}, \mathrm{s}, \mathrm{C}(2)-H), 7.56(2 \mathrm{H}, \mathrm{bs}$, C(4/5)-H), $7.46(2 \mathrm{H}, \mathrm{bs}, \mathrm{C}(4 / 5)-H), 4.74\left(4 \mathrm{H}, \mathrm{t}, J 7.2 \mathrm{~Hz}, \mathrm{NCH}_{2}\right)$, $4.16\left(4 \mathrm{H}, \mathrm{t}, J 7.5 \mathrm{~Hz}, \mathrm{NCH}_{2}\right), 1.78\left(4 \mathrm{H}\right.$, quin, $\left.J 7.5 \mathrm{~Hz}, \mathrm{CH}_{2} \mathrm{CH}_{2}\right)$, $1.19\left(4 \mathrm{H}, \mathrm{q}, J 7.2 \mathrm{~Hz}, \mathrm{CH}_{2} \mathrm{CH}_{2}\right), 0.87\left(6 \mathrm{H}, \mathrm{t}, J 7.2 \mathrm{~Hz}, \mathrm{CH}_{2} \mathrm{CH}_{3}\right)$. ${ }^{13} \mathrm{C}-\mathrm{NMR}\left(62.8 \mathrm{MHz}, \mathrm{D}_{2} \mathrm{O}\right): \delta 135.6(\mathrm{CH}), 123.5(\mathrm{CH}), 122.2$ $(\mathrm{CH}), 49.6\left(\mathrm{CH}_{2}\right), 48.7\left(\mathrm{CH}_{2}\right), 31.1\left(\mathrm{CH}_{2}\right), 18.7\left(\mathrm{CH}_{2}\right)$ and 12.5 $\left(\mathrm{CH}_{3}\right) ; \mathrm{m} / \mathrm{z}$ (ESI): $275.2230\left(\mathrm{M}-\mathrm{HCl}_{2} 7^{+}\right), 138.1145\left(\mathrm{M}-\mathrm{Cl}_{2} 7^{2+}\right)$, $\mathrm{C}_{16} \mathrm{H}_{27} \mathrm{~N}_{4}$ requires 275.2230 , found 275.2228. IR (powder) $v$ : 3377, 3066, 2959, 2873, 2112, 1642, 1560, 1461, 1367, 1336, $1162,1112,1023,946,857,753,637 \mathrm{~cm}^{-1}$.

\subsection{1,3-Bis(1-butylimidazolium-3-yl)propane dichloride}

${ }^{1} \mathrm{H}-\mathrm{NMR}\left(400 \mathrm{MHz}, \mathrm{D}_{2} \mathrm{O}\right): \delta 8.75(2 \mathrm{H}, \mathrm{s}, \mathrm{C}(2)-H), 7.41(4 \mathrm{H}, \mathrm{bs}$, $\mathrm{C}(4 / 5)-H), 4.22\left(4 \mathrm{H}, \mathrm{t}, J 7.2 \mathrm{~Hz}, \mathrm{NCH}_{2}\right), 4.09(4 \mathrm{H}, \mathrm{t}, J 7.2 \mathrm{~Hz}$, $\mathrm{NCH}_{2}$ ), $2.42\left(4 \mathrm{H}\right.$, quin, $\left.J 7.2 \mathrm{~Hz}, \mathrm{CH}_{2} \mathrm{CH}_{2} \mathrm{CH}_{2}\right), 1.74$ (4H, quin, $J$ $\left.7.5 \mathrm{~Hz}, \mathrm{CH}_{2} \mathrm{CH}_{2}\right), 1.22\left(4 \mathrm{H}\right.$, quin, $\left.J 7.5 \mathrm{~Hz}, \mathrm{CH}_{2} \mathrm{CH}_{3}\right), 0.81(6 \mathrm{H}$, $\left.\mathrm{t}, J 7.2 \mathrm{~Hz}, \mathrm{CH}_{2} \mathrm{CH}_{3}\right) .{ }^{13} \mathrm{C}-\mathrm{NMR}\left(62.8 \mathrm{MHz}, \mathrm{D}_{2} \mathrm{O}\right): \delta 135.4(\mathrm{CH})$, $122.8(\mathrm{CH}), 122.3(\mathrm{CH}), 49.6\left(\mathrm{CH}_{2}\right), 46.5\left(\mathrm{CH}_{2}\right), 31.2\left(\mathrm{CH}_{2}\right)$, $29.7\left(\mathrm{CH}_{2}\right), 18.8\left(\mathrm{CH}_{2}\right)$ and $12.7\left(\mathrm{CH}_{3}\right) ; \mathrm{m} / \mathrm{z}(\mathrm{ESI}): 289.2385(\mathrm{M}-$ $\left.\mathrm{HCl}_{2} 7^{+}\right), 145.1225\left(\mathrm{M}-\mathrm{Cl}_{2} 7^{2+}\right), \mathrm{C}_{17} \mathrm{H}_{29} \mathrm{~N}_{4}$ requires 289.2387 , found 289.2385. IR (powder) v: 3381, 3133, 1057, 2959, 2872, 2112, 1704, 1641, 1562, 1459, 1370, 1335, 1160, 1114, 1021, 949, 872, $753,635 \mathrm{~cm}^{-1}$.

\subsection{1,4-Bis(1-butylimidazolium-3-yl)butane dichloride}

${ }^{1} \mathrm{H}-\mathrm{NMR}\left(400 \mathrm{MHz}, \mathrm{D}_{2} \mathrm{O}\right): \delta 8.77(2 \mathrm{H}, \mathrm{s}, \mathrm{C}(2)-H), 7.46(4 \mathrm{H}, \mathrm{bs}$, $\mathrm{C}(4 / 5)-H), 4.20\left(4 \mathrm{H}, \mathrm{t}, J 6.4 \mathrm{~Hz}, \mathrm{NCH}_{2}\right), 4.15(4 \mathrm{H}, \mathrm{t}, J 7.2 \mathrm{~Hz}$, $\left.\mathrm{NCH}_{2}\right), 1.84\left(4 \mathrm{H}\right.$, quin $\left.J 6.4 \mathrm{~Hz}, \mathrm{CH}_{2}\right), 1.76(4 \mathrm{H}, \mathrm{t}, J 7.5 \mathrm{~Hz}$, $\left.\mathrm{CH}_{2} \mathrm{CH}_{2}\right), 1.25\left(4 \mathrm{H}\right.$, quin, $\left.J 7.5 \mathrm{~Hz}, \mathrm{CH}_{2} \mathrm{CH}_{3}\right), 0.85(6 \mathrm{H}, \mathrm{t}, J 7.2$ $\left.\mathrm{Hz}, \mathrm{CH}_{2} \mathrm{CH}_{3}\right) .{ }^{13} \mathrm{C}-\mathrm{NMR}\left(62.8 \mathrm{MHz}, \mathrm{D}_{2} \mathrm{O}\right): \delta 135.2(\mathrm{CH}), 122.6$ $(\mathrm{CH}), 122.1(\mathrm{CH}), 49.5\left(\mathrm{CH}_{2}\right), 48.7\left(\mathrm{CH}_{2}\right), 31.2\left(\mathrm{CH}_{2}\right), 26.3$ $\left(\mathrm{CH}_{2}\right), 18.8\left(\mathrm{CH}_{2}\right)$ and $12.7\left(\mathrm{CH}_{3}\right) ; \mathrm{m} / \mathrm{z}(\mathrm{ESI}): 303.2542(\mathrm{M}-$ $\left.\mathrm{HCl}_{2} 7^{+}\right), 152.1305\left(\mathrm{M}-\mathrm{Cl}_{2} 7^{2+}\right), \mathrm{C}_{18} \mathrm{H}_{31} \mathrm{~N}_{4}$ requires 303.2543 , found 303.2542. IR (powder) v: 3383, 3133, 3064, 2959, 2872, 2106, 1632, 1562, 1462, 1373, 1334, 1160, 1114, 1024, 949, 871, 754, $634 \mathrm{~cm}^{-1}$.

\subsection{1,5-Bis(1-butylimidazolium-3-yl)pentane dichloride}

${ }^{1} \mathrm{H}-\mathrm{NMR}\left(400 \mathrm{MHz}, \mathrm{D}_{2} \mathrm{O}\right): \delta 8.70(2 \mathrm{H}, \mathrm{s}, \mathrm{C}(2)-H), 7.39$ (4H, bs, $\mathrm{C}(4 / 5)-H), 4.13-4.06\left(8 \mathrm{H}, \mathrm{m}, J 6.8,7.2 \mathrm{~Hz}, \mathrm{NCH}_{2}\right), 1.85-1.69$ $\left(8 \mathrm{H}, \mathrm{m}, J 6.8,7.2 \mathrm{~Hz}, \mathrm{CH}_{2}\right), 1.18\left(6 \mathrm{H}, \mathrm{m}, J 6.8,7.6 \mathrm{~Hz}, \mathrm{CH}_{2} \mathrm{CH}_{3}\right.$ and $\left.\mathrm{NCH}_{2} \mathrm{CH}_{2} \mathrm{CH}_{2} \mathrm{CH}_{2}\right), 0.80\left(6 \mathrm{H}, \mathrm{t}, J 7.6 \mathrm{~Hz}, \mathrm{CH}_{3}\right) .{ }^{13} \mathrm{C}-\mathrm{NMR}$ $\left(62.8 \mathrm{MHz}, \mathrm{D}_{2} \mathrm{O}\right): \delta 135.1(\mathrm{CH}), 122.4(\mathrm{CH}), 122.2(\mathrm{CH}), 49.3$ $\left(\mathrm{CH}_{2}\right), 31.2\left(\mathrm{CH}_{2}\right), 28.6\left(\mathrm{CH}_{2}\right), 24.96\left(\mathrm{CH}_{2}\right) 22.12\left(\mathrm{CH}_{2}\right) 18.7$ $\left(\mathrm{CH}_{2}\right)$ and $12.6\left(\mathrm{CH}_{3}\right) ; \mathrm{m} / \mathrm{z}(\mathrm{ESI}): 317.2700\left(\mathrm{M}-\mathrm{HCl}_{2} 7^{+}\right), 159.1383$ $\left(\mathrm{M}-\mathrm{Cl}_{2} 7^{2+}\right), \mathrm{C}_{19} \mathrm{H}_{33} \mathrm{~N}_{4}$ requires 317.2700 , found 317.2700. IR (powder) v: 3375, 3133, 3069, 2958, 2930, 2871, 2112, 1765, 1715, 1637, 1562, 1461, 1376, 1330, 1160, 1114, 1058, 1035, $990,869,753,638 \mathrm{~cm}^{-1}$.

4.22. Bis(1-methyl-2-phenylimidazolium-3-yl)methane dichloride

${ }^{1} \mathrm{H}-\mathrm{NMR}\left(400 \mathrm{MHz}, \mathrm{D}_{2} \mathrm{O}\right): \delta 7.69(4 \mathrm{H}, \mathrm{m}, \mathrm{CH}), 7.53(4 \mathrm{H}, \mathrm{m}$, $\mathrm{NCH}), 7.37(2 \mathrm{H}, \mathrm{m}, \mathrm{CH}), 7.25(4 \mathrm{H}, \mathrm{d}, J 7.6 \mathrm{~Hz}, \mathrm{CH}), 6.41(2 \mathrm{H}, \mathrm{s}$, 
$\left.\mathrm{NCH}_{2} \mathrm{~N}\right), 3.58\left(6 \mathrm{H}, \mathrm{s}, \mathrm{NCH}_{3}\right) .{ }^{13} \mathrm{C}-\mathrm{NMR}\left(62.8 \mathrm{MHz}, \mathrm{D}_{2} \mathrm{O}\right): \delta$ $133.6(\mathrm{CH}), 130.1(\mathrm{CH}), 129.7(\mathrm{CH}), 124.2(\mathrm{CH}), 121.7(\mathrm{CH})$, $118.8(\mathrm{CH}), 58.1\left(\mathrm{CH}_{2}\right)$ and $35.8\left(\mathrm{CH}_{2}\right) ; \mathrm{m} / \mathrm{z}(\mathrm{ESI}): 165.0914(\mathrm{M}-$ $\left.\mathrm{Cl}_{2} \mathrm{2}^{2+}\right), \quad \mathrm{C}_{21} \mathrm{H}_{22} \mathrm{~N}_{4}$ requires 165.0917, found 165.0914. IR (powder) v: 3445, 3396, 3044, 1583, 1501, 1435, 1238, 1169, 1095, 1032, 937, 782, 713, $692 \mathrm{~cm}^{-1}$. Anal. Calcd (\%) for $\mathrm{C}_{21} \mathrm{H}_{22} \mathrm{~N}_{4} \mathrm{Cl}_{2} .2 \mathrm{H}_{2} \mathrm{O}$ : (MW 437.37): C, 57.67; H, 5.99; N, 12.81; Found (\%): C, 57.04; H, 5.88; N, 12.56 .

\subsection{1,2-Bis(1-methyl-2-phenylimidazolium-3-yl)ethane} dichloride

${ }^{1} \mathrm{H}-\mathrm{NMR}\left(400 \mathrm{MHz}, \mathrm{D}_{2} \mathrm{O}\right): \delta 7.92(4 \mathrm{H}, \mathrm{m}, \mathrm{CH}), 7.82(4 \mathrm{H}, \mathrm{s}$, $\mathrm{NCH}), 7.69$ (2H, m, CH), $7.30(4 \mathrm{H}, \mathrm{d}, J 4 \mathrm{~Hz}, \mathrm{CH}), 4.38(4 \mathrm{H}, \mathrm{s}$, $\left.\mathrm{NCH}_{2}\right), 3.56\left(6 \mathrm{H}, \mathrm{s}, \mathrm{NCH}_{3}\right) .{ }^{13} \mathrm{C}-\mathrm{NMR}\left(62.8 \mathrm{MHz}, \mathrm{D}_{2} \mathrm{O}\right): \delta 145.0$ $(\mathrm{CH}), 133.2(\mathrm{CH}), 130.1(\mathrm{CH}), 129.6(\mathrm{CH}), 124.3(\mathrm{CH}), 121.4$ $(\mathrm{CH}), 119.4(\mathrm{CH}), 47.75\left(\mathrm{CH}_{2}\right)$ and $35.6\left(\mathrm{CH}_{3}\right) ; \mathrm{m} / \mathrm{z}(\mathrm{ESI})$ :

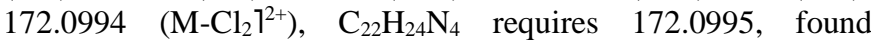
172.0994. IR (powder) v: 3393, 3055, 2103, 1633, 1605, 1582, 1507, 1474, 1439, 1327, 1251, 1218, 1171, 1097, 1026, 938, 776, 708, $602 \mathrm{~cm}^{-1}$. Anal. Calcd (\%) for $\mathrm{C}_{22} \mathrm{H}_{24} \mathrm{~N}_{4} \mathrm{Cl}_{2} \cdot 4 \mathrm{H}_{2} \mathrm{O}$ : (MW 487.43): C, 54.21; H, 6.62; N, 11.49; Found (\%): C, 54.28; H, $6.43 ; \mathrm{N}, 11.36$.

\subsection{1,3-Bis(1-methyl-2-phenylimidazolium-3-yl)propane dichloride}

${ }^{1} \mathrm{H}-\mathrm{NMR}\left(400 \mathrm{MHz}, \mathrm{D}_{2} \mathrm{O}\right): \delta 7.71(4 \mathrm{H}, \mathrm{m}, \mathrm{CH}), 7.60(4 \mathrm{H}, \mathrm{m}$, $\mathrm{NCH}), 7.43(2 \mathrm{H}, \mathrm{s}, \mathrm{CH}), 7.36(4 \mathrm{H}, \mathrm{m}, \mathrm{CH}), 3.86(4 \mathrm{H}, \mathrm{t}, J 7.2 \mathrm{~Hz}$, $\left.\mathrm{NCH}_{2}\right), 3.56\left(6 \mathrm{H}, \mathrm{s}, \mathrm{NCH}_{3}\right)$ and $2.03\left(2 \mathrm{H}\right.$, quin, $\left.J 7.2 \mathrm{~Hz}, \mathrm{CH}_{2}\right)$. ${ }^{13} \mathrm{C}-\mathrm{NMR}\left(62.8 \mathrm{MHz}, \mathrm{D}_{2} \mathrm{O}\right): \delta 132.8(\mathrm{CH}), 129.9(\mathrm{CH}), 129.8$ $(\mathrm{CH}), 123.5(\mathrm{CH}), 121.2(\mathrm{CH}), 120.5(\mathrm{CH}), 45.0(\mathrm{CH}), 35.2$ $\left(\mathrm{CH}_{2}\right)$ and $29.8\left(\mathrm{CH}_{3}\right) ; \mathrm{m} / \mathrm{z}(\mathrm{ESI})$ : $\left.179.1075\left(\mathrm{M}-\mathrm{Cl}_{2}\right]^{2+}\right), \mathrm{C}_{23} \mathrm{H}_{26} \mathrm{~N}_{4}$ requires 179.1145 , found 179.1075 . IR (powder) v: 3481, 3402, 3344, 3234, 3071, 1633, 1580, 1510, 1451, 1330, 1247, 1156, 1085, 945, 786, 787, $715 \mathrm{~cm}^{-1}$. Anal. Calcd (\%) for $\mathrm{C}_{23} \mathrm{H}_{26} \mathrm{~N}_{4} \mathrm{Cl}_{2} .2 \mathrm{H}_{2} \mathrm{O}$ : (MW 465.42): C, 59.36; H, 6.50; N, 12.04; Found (\%): C, 59.18; H, 6.40; N, 11.88 .

\subsection{1,4-Bis(1-methyl-2-phenylimidazolium-3-yl)butane dichloride}

${ }^{1} \mathrm{H}-\mathrm{NMR}\left(400 \mathrm{MHz}, \mathrm{D}_{2} \mathrm{O}\right): \delta 7.68(4 \mathrm{H}, \mathrm{m}, \mathrm{CH}), 7.56(4 \mathrm{H}, \mathrm{m}$, $\mathrm{NCH}), 7.43(2 \mathrm{H}, \mathrm{d}, J 2.0 \mathrm{~Hz}, \mathrm{CH}), 7.38(4 \mathrm{H}, \mathrm{d}, J 6.8 \mathrm{~Hz}, \mathrm{CH})$, $3.80\left(4 \mathrm{H}, \mathrm{t}, J 6.8 \mathrm{~Hz}, \mathrm{NCH}_{2}\right), 3.56\left(6 \mathrm{H}, \mathrm{s}, \mathrm{NCH}_{3}\right)$ and $1.40(4 \mathrm{H}$, quin, $\left.J 6.8 \mathrm{~Hz}, \mathrm{CH}_{2}\right) .{ }^{13} \mathrm{C}-\mathrm{NMR}\left(62.8 \mathrm{MHz}, \mathrm{D}_{2} \mathrm{O}\right): \delta 144.6(\mathrm{CH})$, $132.7(\mathrm{CH}), 129.9(\mathrm{CH}), 129.7(\mathrm{CH}), 123.2(\mathrm{CH}), 121.4(\mathrm{CH})$, $120.8(\mathrm{CH}), 47.4\left(\mathrm{CH}_{2}\right), 35.2\left(\mathrm{CH}_{2}\right)$ and $25.4\left(\mathrm{CH}_{3}\right) ; \mathrm{m} / \mathrm{z}(\mathrm{ESI})$ : $186.11150\left(\mathrm{M}-\mathrm{Cl}_{2} 7^{2+}\right), \quad \mathrm{C}_{24} \mathrm{H}_{28} \mathrm{~N}_{4}$ requires 186.1152 , found 186.1150. IR (powder) v: 3467, 3403, 3047, 3020, 1704, 1619, $1580,1516,1480,1468,1449,1392,1319,1258,1241,1159$, 1113, 1094, 1050, 1004, 953, 813, 784, 719, 707, 618, $555 \mathrm{~cm}^{-1}$. Anal. Calcd (\%) for $\mathrm{C}_{24} \mathrm{H}_{28} \mathrm{~N}_{4} \mathrm{Cl}_{2} .2 \mathrm{H}_{2} \mathrm{O}$ : (MW 479.45): C, 60.12; H, 6.73; N, 11.68; Found (\%): C, 59.76; H, 6.70; N, 11.40.

\subsection{1,5-Bis(1-methyl-2-phenylimidazolium-3-yl)pentane dichloride}

${ }^{1} \mathrm{H}-\mathrm{NMR}\left(400 \mathrm{MHz}, \mathrm{D}_{2} \mathrm{O}\right): \delta 7.66(4 \mathrm{H}, \mathrm{m}, \mathrm{CH}), 7.60(4 \mathrm{H}, \mathrm{m}$, $\mathrm{NCH}), 7.44(2 \mathrm{H}, \mathrm{m}, \mathrm{CH}), 7.39(4 \mathrm{H}, \mathrm{m}, \mathrm{CH}), 3.82(4 \mathrm{H}, \mathrm{t}, J 7.2$ $\left.\mathrm{Hz}, \mathrm{NCH}_{2}\right), 3.57\left(6 \mathrm{H}, \mathrm{s}, \mathrm{NCH}_{3}\right) 1.43\left(4 \mathrm{H}\right.$, quin, $\left.J 7.2 \mathrm{~Hz}, \mathrm{CH}_{2}\right)$ $0.90\left(2 \mathrm{H}\right.$, quin, $\left.J 7.2 \mathrm{~Hz}, \mathrm{CH}_{2}\right) .{ }^{13} \mathrm{C}-\mathrm{NMR}\left(62.8 \mathrm{MHz}, \mathrm{D}_{2} \mathrm{O}\right): \delta$ $144.6(\mathrm{C}), 132.6(\mathrm{CH}), 130.1(\mathrm{CH}), 129.6(\mathrm{CH}), 123.1(\mathrm{CH})$, $121.4(\mathrm{CH}), 121.0(\mathrm{C}), 47.9\left(\mathrm{CH}_{2}\right), 35.2\left(\mathrm{CH}_{3}\right), 28.2\left(\mathrm{CH}_{2}\right)$ and $21.7\left(\mathrm{CH}_{2}\right) ; \mathrm{m} / \mathrm{z}$ (ESI): $193.1230\left(\mathrm{M}-\mathrm{Cl}_{2} 7^{2+}\right), \mathrm{C}_{25} \mathrm{H}_{30} \mathrm{~N}_{4} \mathrm{Cl}_{2} .4 \mathrm{H}_{2} \mathrm{O}$ requires 193.1230, found 193.1230. IR (powder) v: 3503, 3405, 3366, 3118, 3068, 1603, 1580, 1511, 1470, 1444, 1411, 1363, $1328,1245,942,785,777,705,699,619,603 \mathrm{~cm}^{-1}$. Anal. Calcd
(\%) for $\mathrm{C}_{25} \mathrm{H}_{31} \mathrm{~N}_{4} \mathrm{Cl}_{2} .4 \mathrm{H}_{2} \mathrm{O}$ : (MW 529.50): C, 56.71; H, 7.23; N, 10.58; Found (\%): C, 57.02; H, 6.92; N, 10.48.

\subsection{Bis(1,2,4,5-tetramethylimidazolium-3-yl)methane dichloride}

${ }^{1} \mathrm{H}-\mathrm{NMR}\left(400 \mathrm{MHz}, \mathrm{D}_{2} \mathrm{O}\right): \delta 6.36\left(2 \mathrm{H}, \mathrm{s}, \mathrm{NCH}_{2}\right), 3.60(6 \mathrm{H}, \mathrm{s}$, $\left.\mathrm{NCH}_{3}\right), 2.57\left(6 \mathrm{H}, \mathrm{s}, \mathrm{CCH}_{3}\right), 2.14\left(6 \mathrm{H}, \mathrm{s}, \mathrm{CCH}_{3}\right)$ and $1.98(6 \mathrm{H}, \mathrm{s}$, $\left.\mathrm{CCH}_{3}\right) .{ }^{13} \mathrm{C}-\mathrm{NMR}\left(62.8 \mathrm{MHz}, \mathrm{D}_{2} \mathrm{O}\right): \delta 144.0\left(\mathrm{CH}_{2}\right), 128.2\left(\mathrm{CH}_{2}\right)$, $125.3\left(\mathrm{CH}_{2}\right), 54.7\left(\mathrm{CH}_{2}\right), 32.0\left(\mathrm{CH}_{3}\right), 10.0\left(\mathrm{CH}_{3}\right), 7.9\left(\mathrm{CH}_{3}\right)$ and

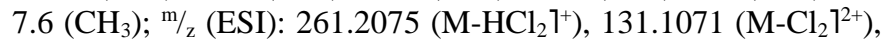
$\mathrm{C}_{15} \mathrm{H}_{25} \mathrm{~N}_{4}$ requires 261.2074, found 261.2075. IR (powder) $v$ : 3446, 3370, 2985, 2121, 1652, 1623, 1542, 1507, 1443, 1396, 1364, 1329, 1239, 1205, 1140, 1077, 1037, 865, 846, 822, 711, 590, $567 \mathrm{~cm}^{-1}$. Anal. Calcd (\%) for $\mathrm{C}_{15} \mathrm{H}_{26} \mathrm{~N}_{4} \mathrm{Cl}_{2} \cdot 3 \mathrm{H}_{2} \mathrm{O}$ : (MW 387.35): C, 46.51; H, 8.33; N, 14.46; Found (\%): C, 45.34; H, $8.38 ; \mathrm{N}, 14.02$.

\subsection{1,2-Bis(1,2,4,5-tetramethylimidazolium-3-yl)ethane dichloride}

${ }^{1} \mathrm{H}-\mathrm{NMR}\left(400 \mathrm{MHz}, \mathrm{D}_{2} \mathrm{O}\right): \delta 4.40\left(4 \mathrm{H}, \mathrm{s}, \mathrm{NCH}_{2}\right), 3.55(6 \mathrm{H}, \mathrm{s}$, $\left.\mathrm{NCH}_{3}\right), 2.31\left(6 \mathrm{H}, \mathrm{s}, \mathrm{CCH}_{3}\right), 2.13\left(6 \mathrm{H}, \mathrm{s}, \mathrm{CCH}_{3}\right), 1.99(6 \mathrm{H}, \mathrm{s}$, $\left.\mathrm{CCH}_{3}\right) .{ }^{13} \mathrm{C}-\mathrm{NMR}\left(62.8 \mathrm{MHz}, \mathrm{D}_{2} \mathrm{O}\right): \delta 142.7(\mathrm{C}), 127.3(\mathrm{C})$, $125.2(\mathrm{C}), 43.8\left(\mathrm{CH}_{2}\right), 31.7\left(\mathrm{CH}_{3}\right), 9.0\left(\mathrm{CH}_{3}\right), 7.6\left(\mathrm{CH}_{3}\right)$ and 7.2

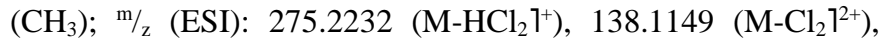
$\mathrm{C}_{16} \mathrm{H}_{27} \mathrm{~N}_{4}$ requires 275.2230, found 275.2232. IR (powder) $v$ : 3427, 3385, 2975, 2117, 1644, 1617, 1541, 1525, 1481, 1442, 1379, 1357, 1339, 1272, 1230, 1182, 1135, 1077, 1047, 851, 835, 768, 667, 613, $564 \mathrm{~cm}^{-1}$. Anal. Calcd (\%) for $\mathrm{C}_{16} \mathrm{H}_{28} \mathrm{~N}_{4} \mathrm{Cl}_{2}$.2.5 $\mathrm{H}_{2} \mathrm{O}$ : (MW 392.37): C, 48.98; H, 8.47; N, 14.28; Found (\%): C, 48.56; H, 8.44; N, 14.17.

\subsection{1,3-Bis(1,2,4,5-tetramethylimidazolium-3-yl)propane dichloride}

${ }^{1} \mathrm{H}-\mathrm{NMR}\left(400 \mathrm{MHz}, \mathrm{D}_{2} \mathrm{O}\right): \delta 4.09\left(4 \mathrm{H}, \mathrm{t}, J 7.1 \mathrm{~Hz}, \mathrm{NCH}_{2}\right), 3.53$ $\left(6 \mathrm{H}, \mathrm{s}, \mathrm{NCH}_{3}\right), 2.53\left(6 \mathrm{H}, \mathrm{s}, \mathrm{CCH}_{3}\right), 2.47\left(6 \mathrm{H}, \mathrm{s}, \mathrm{CCH}_{3}\right), 2.11(6 \mathrm{H}$, $\left.\mathrm{s}, \mathrm{CCH}_{3}\right)$ and $1.08\left(2 \mathrm{H}\right.$, quin, $\left.J 7.1 \mathrm{~Hz} \mathrm{CH} \mathrm{CH}_{2}\right) .{ }^{13} \mathrm{C}-\mathrm{NMR}(62.8$ $\left.\mathrm{MHz}, \mathrm{D}_{2} \mathrm{O}\right): \delta 142.1(\mathrm{C}), 126.4(\mathrm{C}), 124.6(\mathrm{C}), 41.6\left(\mathrm{CH}_{3}\right), 31.2$ $\left(\mathrm{CH}_{2}\right), 28.7\left(\mathrm{CH}_{2}\right), 9.3\left(\mathrm{CH}_{3}\right), 7.6\left(\mathrm{CH}_{3}\right)$ and $7.5\left(\mathrm{CH}_{3}\right) ; \mathrm{m} / \mathrm{z}(\mathrm{ESI})$ : $289.2388\left(\mathrm{M}-\mathrm{HCl}_{2} \mathrm{7}^{+}\right), 145.1227\left(\mathrm{M}-\mathrm{Cl}_{2} 7^{2+}\right), \mathrm{C}_{17} \mathrm{H}_{29} \mathrm{~N}_{4}$ requires 289.2387, found 289.2388. IR (powder) v: 3370, 3284, 2983, 2923, 2110, 1643, 1528, 1430, 1376, 1351, 1227, 1206, 1177, 1064, 1037, 853, 824, 767, 644, 577, $561 \mathrm{~cm}^{-1}$. Anal. Calcd (\%) for $\mathrm{C}_{17} \mathrm{H}_{30} \mathrm{~N}_{4} \mathrm{Cl}_{2} .4 \mathrm{H}_{2} \mathrm{O}$ : (MW 433.42): $\mathrm{C}, 47.11 ; \mathrm{H}, 8.84 ; \mathrm{N}$, 12.93; Found (\%): C, 46.74; H, 8.96; N, 12.68.

\subsection{1,4-Bis(1,2,4,5-tetramethylimidazolium-3-yl)butane dichloride}

${ }^{1} \mathrm{H}-\mathrm{NMR}\left(400 \mathrm{MHz}, \mathrm{D}_{2} \mathrm{O}\right): \delta 3.97\left(4 \mathrm{H}, \mathrm{t}, J 5.0, \mathrm{NCH}_{2}\right), 3.50(6 \mathrm{H}$, s, $\left.\mathrm{NCH}_{3}\right), 2.45\left(6 \mathrm{H}, \mathrm{s}, \mathrm{CCH}_{3}\right), 2.13\left(6 \mathrm{H}, \mathrm{s}, \mathrm{CCH}_{3}\right), 2.13(6 \mathrm{H}, \mathrm{s}$, $\left.\mathrm{CCH}_{3}\right)$ and $1.66\left(4 \mathrm{H}\right.$, quin, $\left.J 5.0 \mathrm{~Hz}, \mathrm{CH}_{2}\right) .{ }^{13} \mathrm{C}-\mathrm{NMR}(62.8 \mathrm{MHz}$, $\left.\mathrm{D}_{2} \mathrm{O}\right): \delta 142.0(\mathrm{C}), 126.5(\mathrm{C}), 124.6(\mathrm{C}), 44.2\left(\mathrm{CH}_{2}\right), 31.2\left(\mathrm{CH}_{3}\right)$, $25.9\left(\mathrm{CH}_{2}\right), 9.3\left(\mathrm{CH}_{3}\right), 7.54\left(\mathrm{CH}_{3}\right), 7.53\left(\mathrm{CH}_{3}\right) ; \mathrm{m} / \mathrm{z}(\mathrm{ESI})$ : $303.2546\left(\mathrm{M}-\mathrm{HCl}_{2} 7^{+}\right), 152.1307\left(\mathrm{M}-\mathrm{Cl}_{2} 7^{2+}\right), \mathrm{C}_{18} \mathrm{H}_{31} \mathrm{~N}_{4}$ requires 303.2543, found 303.2546. IR (powder) v: 3386, 3340, 2986, 2875, 2100, 1649, 1609, 1533, 1448, 1416, 1373, 1224, 1167, $1078, \quad 1042,838,759,692 \mathrm{~cm}^{-1}$. Anal. Calcd (\%) for $\mathrm{C}_{18} \mathrm{H}_{32} \mathrm{~N}_{4} \mathrm{Cl}_{2} .4 \mathrm{H}_{2} \mathrm{O}$ : (MW 447.44): C, 48.32; H, 9.01; N, 12.52; Found (\%): C, 47.40; H, 8.48; N, 12.58.

\subsection{1,5-Bis(1,2,4,5-tetramethylimidazolium-3-yl)pentane dichloride}

${ }^{1} \mathrm{H}-\mathrm{NMR}\left(400 \mathrm{MHz}, \mathrm{D}_{2} \mathrm{O}\right): \delta 3.93\left(4 \mathrm{H}, \mathrm{t}, J 7.6 \mathrm{~Hz}, \mathrm{NCH}_{2}\right), 3.46$ $\left(6 \mathrm{H}, \mathrm{s}, \mathrm{NCH}_{3}\right), 2.48\left(6 \mathrm{H}, \mathrm{s}, \mathrm{CCH}_{3}\right), 2.48\left(6 \mathrm{H}, \mathrm{s}, \mathrm{CCH}_{3}\right), 2.10(6 \mathrm{H}$, $\left.\mathrm{s}, \mathrm{CCH}_{3}\right), 1.73\left(4 \mathrm{H}\right.$, quin, $\left.J 7.6 \mathrm{~Hz}, \mathrm{CH}_{2}\right)$ and $1.26(2 \mathrm{H}, \mathrm{q}, J 7.6$ $\left.\mathrm{Hz}, \mathrm{CH}_{2}\right) .{ }^{13} \mathrm{C}-\mathrm{NMR}\left(62.8 \mathrm{MHz}, \mathrm{D}_{2} \mathrm{O}\right): \delta 141.9(\mathrm{C}), 125.8(\mathrm{C})$, 
$124.7(\mathrm{C}), 45.1\left(\mathrm{CH}_{2}\right), 31.1\left(\mathrm{CH}_{3}\right), 28.5\left(\mathrm{CH}_{2}\right), 22.9\left(\mathrm{CH}_{2}\right), 9.2$

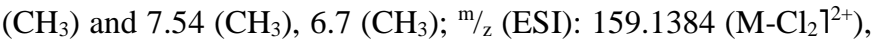
$\mathrm{C}_{19} \mathrm{H}_{34} \mathrm{~N}_{4}$ requires 159.1386 , found 159.1384 . IR (powder) $v$ : 3368, 2927, 2865, 2116, 1703, 1645, 1531, 1433, 1373, 1227, $1132,1076,1046,863,839,739,599 \mathrm{~cm}^{-1}$.

\section{Acknowledgments}

Use of the University of Reading Chemical Analysis Facility is gratefully acknowledged. D. L. S. is grateful to the Portuguese Foundation for Science and Technology for funding (ref SFRH/BD/36590/2007). P. O. P. is grateful to the University of Reading for funding.

\section{References}

1. Kreher, U. P.; Rosamilia, A. E.; Raston, C. L.; Scott, J. L.; Strauss, C. R. Molecules 2004, 9, 387-393.

2. Jin, C.; Wu, L.; Han, D.; Hu, Y. Acta Crys. 2006, E62, o561905620.

3. Chiou, J. Y. Z.; Chen, J. N.; Lei, J. S.; Lin, I. J. B. J. Mater. Chem. 2006, 16, 2972-2977.

4. Baleizão, C.; Gigante, B.; Garcia, H.; Corma, A. Green Chem. 2002, 4, 272-274.

5. Kornilov, D. A.; Kiselev, V. D.; Konovalov, A. I. Rus. Chem. Bull. 2017, 66(3): 564-566.

6. Benson, J.; Berson, J. J. Am. Chem. Soc. 1962, 84(2), 152-158.

7. Schettino, V.; Bini, R. Chem. Soc. Rev. 2007, 36(6), 869-880.

8. Hamann, S. Rev. Phys. Chem. Japan 1980, 50(147), 147-168.

9. Jenner, G. Mini. Rev. Org. Chem. 2004, 1(1), 9-26.

10. Gonikberg, M.; Zhulin, V. Aust. J. Chem. 1958, 11(3), 285-289.

11. Gonikberg, M.; El'yanov B. Bull. Acad. Sci. USSR 1960, 9(4), 597-602.

12. Holzapfel, W.; Isaacs, N. High-Pressure Techniques in Chemistry and Physics, a Practical Approach, Oxford University Press, Oxford, 1997

13. Hansen, S.; Nordhold L. J. Chromatogr. 1981, 204, 91-101.

14. Wright, D.; Wulff, C. J. Org. Chem. 1970, 35(12), 4252-4252.

15. Mills, J.; Maryanoff, C.; McComsey, D.; Stanzione, R.; Scott, L. J. Org. Chem. 1987, 52(9), 1857-1859.

16. Rudine, A.; Walter, M.; Wamser, C. J. Org. Chem. 2010, 75(12), 4292-4295.

17. Wright D.; Wulff C. J. Org. Chem. 1970, 35(12), 4252.

18. Beckett, A.; Ali, H. J. Chromatogr. 1979, 177(2), 91-101.

19. Zhou, F.; Liang, Y.; Liu, W. Chem. Soc. Rev. 2009, 38(9), 25902599.

20. Somers, A.; Howlett, P.; MacFarlane, D.; Forsyth, M. Lubricants 2013, 1(1), 3-21.

21. Yao, M.; Liang, Y.; Xia, Y.; Zhou, F. ACS Appl. Mater. Interfaces 2009, 1(2), 467-471.

22. Menger, F.; Littau, C. J. Am. Chem. Soc. 1993, 115(22), 10083 10090.

23. Ding, Y. S.; Zha, M.; Zhang, J.; Wang, S. Colloids Surf. A Physicochem. Eng. Asp. 2007, 298(3), 201-205.

24. Matsumoto, K.; Hashimoto, S.; Ikemi, Y.; Otani, S. Heterocycles 1984, 22(6), 1417-1420.

25. Almarzoqi, B.; George, A.; Isaacs, N. Tetrahedron 1986, 42(2), 601-607.

26. Le Noble, W.; Asano, T. J. Am. Chem. Soc. 1975, 97(7), 17781782.

27. Berg U.; Chanon M.; Gallo R.; Rajzmann M. J. Org. Chem. 1995, 60(7), 1975-80.

28. Drude, P.; Nernst, W. Z. Phys. Chem. 1894, 15(1), 79-83.

29. Cheung C. K.; Wedinger, R. S.; Le Noble, W. J. J. Org. Chem. 1989, 54(3), 570-573.

30. Arnett, E. M.; Reich, R. J. Am. Chem. Soc. 1980, 102(18), 5892 5902.

31. Gacem, B.; Jenner, G. J. Phys. Org. Chem. 2004, 17(3), 221-225. 